\title{
Receptivity of inviscid modes in supersonic boundary layers to wall perturbations
}

\author{
Yinhui Liu · Ming Dong · Xuesong Wu(D
}

Received: 23 October 2020 / Accepted: 15 March 2021 / Published online: 2 June 2021

(C) The Author(s) 2021

\begin{abstract}
The present paper investigates the receptivity of inviscid first and second modes in a supersonic boundary layer to time-periodic wall disturbances in the form of local blowing/suction, streamwise velocity perturbation and temperature perturbation, all introduced via a small forcing slot on the flat plate. The receptivity is studied using direct numerical simulations (DNS), finite- and high-Reynolds-number approaches, which complement each other. The finite-Reynolds-number formulation predicts the receptivity as accurately as DNS, but does not give much insight to the detailed excitation process, nor can it explain the significantly weaker receptivity efficiency of the streamwise velocity and temperature perturbations relative to the blowing/suction. In order to shed light on these issues, an asymptotic analysis was performed in the limit of large Reynolds number. It shows that the receptivity to all three forms of wall perturbations is reduced to the same mathematical form: the Rayleigh equation subject to an equivalent suction/blowing velocity, which can be expressed explicitly in terms of the physical wall perturbations. Estimates of the magnitude of the excited eigenmode can be made a priori for each case. Furthermore, the receptivity efficiencies for the streamwise velocity and temperature perturbations are quantitatively related to that for the blowing/suction by simple ratios, which are of $O\left(R^{-1 / 2}\right)$ and have simple expressions, where $R$ is the Reynolds number based on the boundary-layer thickness at the centre of the forcing slot. The simple leading-order asymptotic theory predicts the instability and receptivity characteristics accurately for sufficiently large Reynolds numbers (about $10^{4}$ ), but appreciable error exists for moderate Reynolds numbers. An improved asymptotic theory is developed by using the appropriate impedance condition that accounts for the $O\left(R^{-1 / 2}\right)$ transverse velocity induced by the viscous motion in the Stokes layer adjacent to the wall. The improved theory predicts both the instability and receptivity at moderate Reynolds numbers $\left(R=O\left(10^{3}\right)\right)$ with satisfactory accuracy. In particular, it captures well the finiteReynolds-number effects, including the Reynolds-number dependence of the receptivity and the strong excitation occurring near the so-called synchronisation point.
\end{abstract}

Y. Liu

COMAC Beijing Aircraft Technology Research Institute, Beijing 102211, China

Y. Liu · M. Dong

Laboratory of High-speed Aerodynamics, Tianjin University, Tianjin 300072, China

$\mathrm{X}$. Wu $(\square)$

School of Mechanical Engineering, Nantong University, Nantong 226019, China

e-mail: x.wu@ic.ac.uk

$\mathrm{X} . \mathrm{Wu}$

Department of Mathematics, Imperial College London, 180 Queen's Gate, London SW7 2AZ, UK 
Keywords Boundary-layer stability $\cdot$ Receptivity $\cdot$ Transition to turbulence

\section{Introduction}

Transition prediction of boundary layer flows from a laminar to turbulent state is of critical technological importance, especially for the development of super/hypersonic vehicles that are to be used for deep space access [1,2]. The intrinsic cause of transition is the flow instabilities, which include, according to their dominant physical mechanisms, shear instability [3] and centrifugal (Görtler) instability over concave surfaces [4,5] and attachment-line instability [6]. Shear instability may further be subdivided into viscous and inviscid instabilities; crossflow instability in a threedimensional boundary layer belongs to shear instability [7], and is of inviscid nature in its developed phase [8], but turns out to be viscous in its genesis stage near the leading edge [9]. Instabilities in most situations may be formulated mathematically as eigenvalue problems, which are solved to identify the eigenmodes (i.e. the disturbances most likely to amplify) and further to predict their characteristic frequencies and wavelengths. However, transition is also strongly influenced by external factors, i.e. disturbances in the environment such as acoustic, vortical and entropy fluctuations in the free stream, and surface perturbations (e.g. roughness and gaps). In an environment of weak disturbances, transition takes place through three stages: receptivity, linear eigenmode growth and non-linear breakdown to turbulence [10-12]. In the receptivity process, external perturbations excite instability modes of the boundary layer $[13,14]$. Receptivity study aims to identify the mechanisms by which external perturbations excite intrinsic instability modes thereby determining the initial amplitudes and phases of the latter. Mathematically, the principal condition for effective excitation is the scale tuning, that is, external disturbances have to produce a forcing with the length and time scales matching with those of the eigenmodes $[15,16]$.

Boundary-layer flows were introduced and described in an asymptotic framework based upon the high-Reynoldsnumber assumption. Asymptotic techniques have been instrumental in revealing the shear instability and the associated receptivity in these flows. It was through developing and utilising the asymptotic techniques [17-19] that a shear flow with a non-inflectional velocity profile, such as the plane Poiseuille flow and the Blasius boundary layer, was shown to support viscous instability, referred to as Tollmien-Schlichting (T-S) instability, and further advance of the techniques allowed the instability characteristics to be reliably calculated [20,21]. In these earliest studies, which predated the era of digital computer, the asymptotic methods were the only means to find approximate solutions to the instability, i.e. Orr-Sommerfield (O-S), equation. With the advent of modern digital computer and efficient algorithms since the 1970s, the O-S equation, as well as its extensions to compressible and/or three-dimensional flows, can be routinely solved numerically with great accuracy. Yet the value of the asymptotic methods has not been diminished. This is because in the $\mathrm{O}-\mathrm{S}$ type of equations, the instability modes are, explicitly or implicitly, taken to have wavelengths and phase speeds comparable with the boundary-layer thickness and the base-flow velocity, respectively. Then the viscous diffusion and non-parallel-flow effect both appear to be of $O\left(R^{-1}\right)$, yet the latter is neglected while the former is retained in order for instability to be possible at all. The instability thus appears rather unconvincing since it looks like an outcome of retaining, in an ad hoc manner, the small viscous effect, which is as feeble as the non-parallelism.

The relative importance of viscosity and non-parallelism has been quantified by the high-Reynolds-number asymptotic analysis, which shows that the characteristic wavelength of the viscous instability is much longer than the local boundary-layer thickness [20]. In the lower-branch regime, the correct characteristic wavelength is of $O\left(R^{-3 / 8} L\right)$ [20], where $R$ is the Reynolds number based on the distance $L$ to the leading edge. As a result of this scaling and ensuing triple-deck structure, the viscous diffusion in the wall-normal direction is a leading-order effect (which gives rise to the instability), whereas the non-parallel-flow terms merely provide a small $O\left(R^{-3 / 8}\right)$ correction [22]. As well as justifying the ad hoc approximations leading to the $\mathrm{O}-\mathrm{S}$ type of formulations, the asymptotic analysis has identified the essential physical mechanism whereby offering a convincing rational explanation of the viscous T-S instability. The asymptotic descriptions have been provided for instabilities of supersonic [23-25] and threedimensional $[9,26]$ boundary layers. The precise asymptotic scalings and structures of the instability modes are crucial for investigations of receptivity and non-linear evolution [12]. 
Substantially progress on boundary-layer receptivity has been made primarily through detailed asymptotic analyses. In the incompressible or subsonic regime, several mechanisms of boundary-layer receptivity to free-stream disturbances have been identified and described by using high-Reynolds-number asymptotic approach. The first is referred to as the leading-edge adjustment. An incident sound wave impinges on the rapidly changing boundary layer flow near the leading edge to excite first the so-called Lam-Rott asymptotic eigenmode. The latter then undergoes wavelength shortening to evolve into a lower-branch viscous T-S instability mode [27]. This mechanism is however rather weak because the Lam-Rott mode decays exponentially before reaching the lower-branch neutral position, as a result of which the initial amplitude of the excited T-S wave is transcendentally small. The effect of the leading-edge bluntness, which is present in any real aerodynamic body, has been investigated and found to reduce the receptivity $[28,29]$.

The second and more efficient mechanism is the local acoustic receptivity. A free-stream sound wave generates in the boundary layer an unsteady fluctuation, which interacts with the mean-flow distortion induced by a local roughness $[15,16]$. The resulting time-periodic forcing satisfies the scaling matching condition if the frequency of the sound wave and the length scale of the roughness are comparable, respectively, with those of the lowerbranch instability. Interestingly, if the sound wave amplitude is $\epsilon_{s}$ and the roughness height normalised by the local boundary-layer thickness is $h \ll 1$, the amplitude of the instability mode excited is $O\left(\epsilon_{s} h R^{1 / 8}\right)$ rather than $O\left(\epsilon_{S} h\right)$ as one might intuitively expect. A free-stream vortical disturbance, with amplitude $\epsilon_{v}$ say, interacts with a similar roughness also to excite a T-S mode but with an amplitude of $O\left(\epsilon_{v} h\right)[30,31]$. A vortical or acoustic disturbance interacts with distributed roughness [32], generating T-S modes with amplitudes of $O\left(\epsilon_{s} h R^{5 / 16}\right)$ and $O\left(\epsilon_{v} h R^{3 / 16}\right)$, respectively. A T-S mode may also be excited in a flat-plate boundary layer by the interaction between a pair of suitable free-stream vortical and acoustic disturbances [33]. The a priori estimates of the amplitudes, the appropriate specification of the vortical disturbance, and in the case of distributed roughness the description of the resonance between the forcing and the eigenmode [32,33] all depend on the asymptotic approach. The accuracy of some of the asymptotic predictions was assessed by comparison with DNS results [34] as well as with experimental data $[31,32]$, and the quantitative agreement turned out to be rather good or reasonable. Asymptotic theories have been developed for the receptivity to wing surface vibration [35] as well as for the non-linear receptivity due to scattering of a sound wave by a localised roughness element in the transonic regime [36].

As an alternative to the asymptotic approach, a finite-Reynolds-number formulation based on the inhomogeneous $\mathrm{O}-\mathrm{S}$ equation was developed for local receptivity $[37,38]$. While this method was found to give accurate numerical results, it is unable to provide the insights and a priori information that the asymptotic approach offers.

Compared with the subsonic counterpart, the receptivity of supersonic and hypersonic boundary layers becomes more complex $[39,40]$ because there exists a multitude of modes with different physical and mathematical characteristics [41,42]. Among those, the lower-branch first modes with obliqueness angles $\Theta>\sqrt{M^{2}-1}$ are of viscous nature and governed by the triple-deck structure [24], while the first mode with $\Theta<\sqrt{M^{2}-1}$ and the second mode are essentially inviscid and governed by the Rayleigh equation. At Mach numbers greater than 4, the second mode is often more unstable than the first- and higher-order modes, and can play a dominant role in triggering natural transition. Supersonic boundary-layer receptivity exhibits new features, and new mechanisms may come into play.

By using the asymptotic techniques, the slow and fast acoustic waves in the free stream, which propagate upstream and downstream relative to the background flow, respectively, were found to match with the slow and fast boundary-layer eigenmodes, respectively, in the leading-edge region, exciting the latter directly [43-45]. Since the modes generated subsequently undergo little or no attenuation, this leading-edge adjustment mechanism is much more effective than its subsonic counterpart [27,43]. Its operation is restricted though to planar or moderately three-dimensional modes with obliqueness angles $\Theta<\sqrt{M^{2}-1}$, in which the theoretical predictions were found to be in reasonable agreement with experimental measurements [46]. Numerous DNS performed have accumulated a large set of data [47-52], but unfortunately detailed quantitative comparisons with theory are yet to be carried out. The leading-edge receptivity mechanisms for strongly three-dimensional (viscous) modes with $\Theta>\sqrt{M^{2}-1}$ and $\Theta \approx \sqrt{M^{2}-1}$ were discussed by [53] and [54], respectively. 
The local and distributed receptivity due to sound-roughness interaction operates in a similar way to the subsonic regime. However, for the first viscous mode, significant new features arise for free-stream sound waves with frequencies and wavelengths on the triple-deck scales. Such a sound wave with $O\left(\epsilon_{s}\right)$ amplitude generates a much larger $O\left(\epsilon_{s} R^{1 / 8}\right)$ viscous signature in the boundary layer, and as a result the receptivity to it is much stronger than to usual sound waves as was demonstrated by [55] and [56] in the cases of local and distributed roughness, respectively. Moreover, two such sound waves may interact to excite a viscous first mode in a flat-plate boundary layer [56]. For local receptivity of inviscid modes due to sound-roughness interaction, the leading-order forcing arises from the deformation of the acoustic signature by the locally curved boundary, which is purely a surface geometric effect, while the interaction in the bulk of the flow between the acoustic signature and the mean-flow distortion plays a secondary role [57].

In the super- and hyper-sonic regimes, the bow shock may significantly affect the receptivity. Any one of three elementary (acoustic, entropy and vortical) free-stream disturbances interacts with the shock generating all three downstream [58-61]. The receptivity in the presence of a shock has been investigated primarily using DNS in [49-51,62], among others, with the focus being primarily on the leading-edge adjustment. For the boundary layer over a cone, the DNS of the receptivity to acoustic waves suggest that the slow acoustic waves and entropy-layer disturbances after a bow shock play a key role for the excitation of first and second modes, respectively [63,64]. However, the precise scale tuning mechanisms are yet to be identified, which necessarily requires careful asymptotic analyses. A shock close to the boundary layer may cause new instability modes to exist, as it does in the hypersonic boundary layer over a wedge with a small half angle [65]. Interestingly and uniquely, with suitable frequency and wavelength a single (acoustic, vortical or entropy) disturbance upstream of the shock can alone excite this mode near its lower-branch neutral point [66].

Instability modes can be excited by simple forms of external forcing imposed on the surface. In the incompressible limit, the receptivity to time-harmonic blowing/suction, which models a surface vibrating ribbon, was studied in a finite-Reynolds-number formulation $[67,68]$. A T-S mode is excited provided that frequency is in the instability band and the slot width is comparable with, or shorter than, the characteristic wavelength of the instability. This scale matching requirement was demonstrated by using large- $R$ triple-deck formalism [69-71]. The receptivity of supersonic boundary layers to wall perturbations is also important, not least because surface-actuated disturbances, in particular blowing/suction, may be used to control boundary-layer transition. The receptivity of the hypersonic boundary layer over a flat plate to local wall disturbances was studied using finite- $R$ theory [72] and DNS [73], respectively. The boundary layer is found to be much more sensitive to blowing/suction than to the local streamwise velocity and temperature perturbations on the wall, but no explanation was given. Strong excitation occurred when the forcing is near the synchronisation point of the first and second modes. Through asymptotic analysis, the leading-order receptivity of inviscid modes due to the scattering of free-stream sound by localised wall roughness is shown to be equivalent to the receptivity to time-periodic blowing/suction, with the effective or virtual blowing/suction velocity being expressed explicitly in terms of the roughness shape [57]. Blowing/suction thus stands as an elementary building block of more complicated receptivity processes. The present paper will investigate instead the receptivity to three forms of physical wall perturbations, local blowing/suction, streamwise velocity and temperature perturbations, by combining the finite- $R$ approach, DNS and large- $R$ asymptotic analysis, which are complementary to each other. The asymptotic analysis characterises the detailed process of excitation, which amounts, in the present paper, to revealing how the receptivity to the second and third forms of wall perturbations quantitatively link with that to blowing/suction, the most elementary process. On the other hand, DNS and the finite- $R$ approach serve to assess the accuracy of the asymptotic analysis. The present relatively simple problem is used as a vehicle to demonstrate that the important role that asymptotic methods will continue to play in scientific researches.

The rest of the paper is organised as follows. In Sect. 2, the problem is formulated by introducing the key nondimensional parameters and specifying relevant scalings. The finite- $R$ theory and the set-up of DNS are described in Sects. 3 and 4, respectively. In Sect. 5, an asymptotic analysis of the receptivity process is performed to determine the 'initial' amplitude of the instability mode excited and moreover to establish the relations between the three cases. An improved large- $R$ approach is developed to extend the accuracy of the asymptotic theory to moderate 


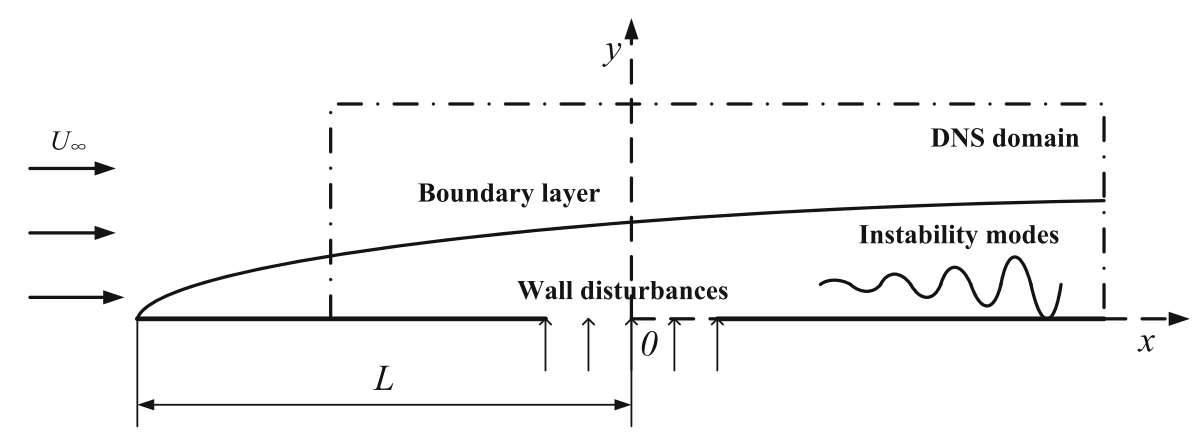

Fig. 1 Sketch of the physical model. The wall disturbances can be three-dimensional, and a cross section in the $x-y$ plane is illustrated

Reynolds numbers. In Sect. 6, we describe the DNS results and carry out comparisons between the theoretical and numerical predictions. A summary and conclusions are given in Sect. 7.

\section{Description of physical problems}

As is shown in Fig. 1, the physical model to be studied is a two-dimensional (2D) supersonic boundary layer formed over a flat plate. The fluid is assumed to be perfect gas, and its viscosity satisfies Sutherland's law. Over a small section of the wall at a distance $L$ downstream of the leading edge, a three-dimensional (3D) spanwise periodic and time-harmonic wall disturbance is introduced, which may take one of the three forms: (1) blowing and suction, (2) streamwise wall motion and (3) temperature fluctuation.

The characteristic boundary-layer thickness at the location of excitation can be defined as $\delta=\sqrt{v_{\infty} L / U_{\infty}}$, where the subscript $\infty$ denotes the quantities of the oncoming flow, and $U$ and $v$ represent the velocity and the kinetic viscosity, respectively. By taking $\delta$ and $U_{\infty}$ as the reference length and velocity, respectively, the Cartesian coordinate system $\left(x^{*}, y^{*}, z^{*}\right)$, with its origin located at the centre of the excitation section, and the time $t^{*}$ are normalised as

$(x, y, z)=\frac{\left(x^{*}, y^{*}, z^{*}\right)}{\delta}$ and $t=\frac{t^{*} U_{\infty}}{\delta}$,

where the asterisk signifies a dimensional quantity. The velocity field, density, temperature and pressure are normalised by their free-stream quantities,

$(u, v, w, \rho, T, p)=\left(\frac{u^{*}}{U_{\infty}}, \frac{v^{*}}{U_{\infty}}, \frac{w^{*}}{U_{\infty}}, \frac{\rho^{*}}{\rho_{\infty}}, \frac{T^{*}}{T_{\infty}}, \frac{p^{*}-p_{\infty}^{*}}{\rho_{\infty} U_{\infty}^{2}}\right)$.

We define the oncoming Mach number $M$ and the Reynolds number $R$ as

$M=\frac{u_{\infty}}{a_{\infty}}, \quad R=\frac{U_{\infty} \delta}{v_{\infty}}=\sqrt{\frac{U_{\infty} L}{v_{\infty}}}$,

where $a_{\infty}$ is the sound speed in the free stream. We are interested in the supersonic regime with $M>1$, and assume that $R \gg 1$ when performing asymptotic analysis.

In this paper, we restrict our attention to the inviscid modes, which have dimensional phase speeds and frequencies of $O\left(U_{\infty}\right)$ and $O\left(U_{\infty} / \delta\right)$, respectively, and their wavelengths are thus comparable with the local boundary-layer thickness $\delta$. The characteristic (streamwise and spanwise) lengths and the frequency of the wall perturbation are taken to be of $O(\delta)$ and $O\left(U_{\infty} / \delta\right)$ as well in order to satisfy the 'resonance' or 'scale matching' condition, which is required for effective generation of instability modes.

For simplicity, the spanwise distribution of the wall disturbance is taken to be sinusoidal. Due to the linearity of the disturbance, it suffices to consider a single Fourier component in the wall perturbation,

$\frac{F(x)\left[\mathrm{e}^{\mathrm{i}(\beta z-\omega t)}+\text { c.c. }\right]}{2}=F(x) \cos (\beta z-\omega t)$, 
where $\beta$ and $\omega$ stand for the spanwise wavenumber and frequency, respectively, and $F(x)$ is the streamwise shape function normalised by its peak value (i.e. $\max |F(x)|=1$ ).

\section{A finite-Reynolds-number theory}

When the non-parallelism of the base flow, which is too weak to be important in receptivity, is neglected, and the amplitude of the wall perturbations is sufficiently small, the $\mathrm{O}-\mathrm{S}$ equation system can be employed to describe the induced unsteady disturbance in the boundary layer including the excitation of eigenmodes $[37,38]$. This is a popular but ad hoc approximation because, as is explained in the introduction, it retains the $O\left(R^{-1}\right)$ viscous effect while neglecting the base-flow non-parallelism of the same order.

The flow field can be decomposed into the background base flow and a time-periodic perturbation, namely,

$\boldsymbol{\phi}=\boldsymbol{\phi}_{\mathrm{B}}+\frac{1}{2} \epsilon_{w} \tilde{\boldsymbol{\phi}}(x, y) \mathrm{e}^{\mathrm{i}(\beta z-\omega t)}+$ c.c.,

where $\boldsymbol{\phi}(y)=\left(u, u_{y}, v, p, T, T_{y}, w, w_{y}\right)^{\mathrm{T}}$ with the subscript B indicating the base flow, $\tilde{\boldsymbol{\phi}}$ represents the excited perturbation, and $\epsilon_{w} \ll 1$ measures the amplitude of the wall perturbations. The unsteady disturbance can be obtained by Fourier transform with respect to $x$, defined as

$\mathscr{F}[\cdot](k) \equiv \frac{1}{\sqrt{2 \pi}} \int_{-\infty}^{\infty} \cdot \exp (-\mathrm{i} k x) \mathrm{d} x$,

where the dot represents a function in physical space and $k$ the streamwise wavenumber. After substituting (3) into the compressible Navier-Stokes (N-S) equations, and neglecting the non-parallelism of the base flow, the Fourier transformed perturbation is found to satisfy the linear system,

$\boldsymbol{L}_{O S}\left(d_{y} ; \omega, k, \beta, R\right) \hat{\boldsymbol{\phi}}_{k}(k, y)=0$,

where $\hat{\boldsymbol{\phi}}_{k}(k, y)=\mathscr{F}[\tilde{\boldsymbol{\phi}}(x, y)]$ and $\boldsymbol{L}_{O S}$ is an $8 \times 8$ matrix O-S operator, which is given in [57] and is equivalent to $\boldsymbol{I} d_{y}-\boldsymbol{A}$ in [74]. The far-field boundary condition is

$\left(\hat{u}_{k}, \hat{v}_{k}, \hat{w}_{k}, \hat{\theta}_{k}\right) \rightarrow 0$ as $y \rightarrow \infty$,

where $\hat{\theta}$ denotes the temperature disturbance. For blowing/suction, the wall boundary condition is

$\hat{u}_{k}=\hat{w}_{k}=\hat{\theta}_{k}=0, \quad \hat{v}_{k}=\hat{F}(k)$ at $y=0$;

for streamwise velocity perturbation, the wall boundary condition is

$\hat{v}_{k}=\hat{w}_{k}=\hat{\theta}_{k}=0, \quad \hat{u}_{k}=\hat{F}(k)$ at $y=0$;

and for temperature perturbation, the wall boundary condition is

$\hat{u}_{k}=\hat{v}_{k}=\hat{w}_{k}=0, \quad \hat{\theta}_{k}=\hat{F}(k)$ at $y=0$.

The solution for $\tilde{\varphi}$ in physical space is obtained by performing inverse Fourier transform,

$\tilde{\varphi}(x, y) \equiv \mathscr{F}^{-1}\left[\hat{\varphi}_{p}(k, y)\right]=\frac{1}{\sqrt{2 \pi}} \int_{-\infty}^{\infty} \hat{\varphi}(k, y) \mathrm{e}^{\mathrm{i} k x} \mathrm{~d} k$.

The integrand has poles at $k=\alpha$, where $\alpha$ stands for the complex streamwise wavenumber of an eigenmode of the homogeneous $\mathrm{O}-\mathrm{S}$ system. We will focus on the poles representing the essentially inviscid modes, which are distinguished by their relatively large phase speeds. Using the residue theorem, we obtain the asymptotic perturbation in the downstream limit $[15,37,38]$

$\tilde{\boldsymbol{\phi}}(x, y) \rightarrow \frac{\sqrt{2 \pi} \mathrm{i}}{\left[\partial\left(\hat{\boldsymbol{\phi}}_{k}\right)^{-1} / \partial k\right]_{k=\alpha}} \mathrm{e}^{\mathrm{i} \alpha x}$ as $x \rightarrow \infty$, 
where the notation $\left(\hat{\boldsymbol{\phi}}_{k}\right)^{-1}$ for a vector $\hat{\boldsymbol{\phi}}_{k}$ is a vector with each component being the reciprocal of the corresponding

component in $\hat{\boldsymbol{\phi}}_{k}$; the same convention applies to the right-hand side of (11), specifically $\left\{\left[\partial\left(\hat{\boldsymbol{\phi}}_{k}\right)^{-1 / \partial k]_{k=\alpha}}\right\}^{-1}\right.$. The boundary-value problem of (5) with (6) and (7) or (8) and (9) is solved using Gaussian elimination for two values of $k$ close to $\alpha$. Then $\left[\partial\left(\hat{\boldsymbol{\phi}}_{k}\right)^{-1} / \partial k\right]_{k=\alpha}$ is calculated using a finite-difference approximation. The present procedure is a straightforward extension of that in $[37,38]$ to supersonic boundary layers by using our eigenvalue solver.

The right-hand side of (11) represents the excited eigenmode, with the pre-factor of $\mathrm{e}^{\mathrm{i} \alpha x}$ being interpreted as the equivalent amplitude at the wall-perturbation centre. The amplitude of the streamwise velocity (scaled by $\epsilon_{w}$, the wall-perturbation intensity) is taken as a measure of the amplitude of the mode excited, denoted by

$\mathscr{A}=\lim _{x \rightarrow \infty} \max _{y} \tilde{u}(x, y) \mathrm{e}^{-\mathrm{i} \alpha x}$.

Since the boundary conditions (7)-(9) are proportional to $\hat{F}(k), \hat{\boldsymbol{\phi}}_{k}$ and hence $\tilde{u}$ depend on $\hat{F}(k)=\hat{F}(\alpha)$ linearly, and we can express the receptivity coefficient as

$\Lambda=\mathscr{A} / \hat{F}(\alpha)$,

which is independent of the distribution function of the wall perturbation.

In the finite-Reynolds-number framework, the receptivity problems for three types of wall perturbations correspond to different boundary conditions, (7)-(9), and thus look all different; no connection can be established between them. On the other hand, the amplitude of the mode excited in all three cases appears to be $O\left(\epsilon_{w}\right)$. However, this turns out to be untrue as will be shown by our high-Reynolds-number asymptotic analysis, which will establish simple quantitative relations between the receptivity coefficients for the three cases under consideration.

\section{Direct numerical simulations (DNS)}

In order to assess the accuracy of the finite- $R$ and large- $R$ theories, we also perform DNS by using our in-house code, which has been applied to a few numerical studies of the transition process $[75,76]$.

The computational domain is chosen to be a rectangular box $x \in\left[x_{0}, x_{I}\right], y \in\left[0, y_{J}\right]$ and $z \in\left[0, z_{K}\right]$. The inflow and outflow boundaries are taken to be at $x_{0}=-200$ and $x_{I}=1000$, respectively, which are sufficiently distant to the location of excitation so that the inhomogeneity induced by the wall perturbation is negligible there. The upper boundary is chosen to be located at $y_{J}=300$, which is in the free stream.

For blowing/suction, the velocity and temperature at the surface satisfies the boundary condition

$[u, v, w, T](x, y, z, t)=\left[0, \epsilon_{w} F(x) \cos (\beta z-\omega t), 0, T_{w}\right] \quad$ at $\quad y=0$.

In the case of a streamwise velocity perturbation, the boundary condition reads

$[u, v, w, T](x, y, z, t)=\left[\epsilon_{w} F(x) \cos (\beta z-\omega t), 0,0, T_{w}\right] \quad$ at $\quad y=0$.

For wall temperature perturbation, the boundary condition becomes

$[u, v, w, T](x, y, z, t)=\left[0,0,0, T_{w}+\epsilon_{w} F(x) \cos (\beta z-\omega t)\right] \quad$ at $\quad y=0$.

Calculations will be performed with a streamwise Gaussian shape,

$F(x)=\mathrm{e}^{-x^{2} / d^{2}}$,

where $d$ measures the streamwise extend of the imposed wall perturbation.

The base flow is taken to be the compressible Blasius similarity solution. Its non-parallelism is of $O\left(R^{-1}\right)$, thus secondary for the receptivity problem and neglected, consistent with the approximation leading to the O-S equations. Accordingly, in DNS we introduce a suitable 'body force' to render the base flow to satisfy the steady N-S equations, a common treatment in a number of previous studies, e.g. [76]. This 'body force' is not arbitrary nor does 


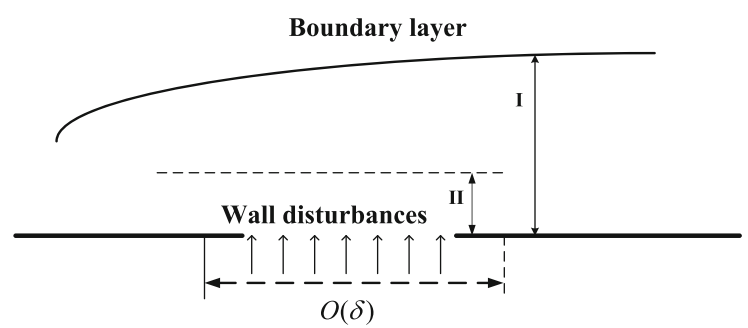

Fig. 2 Asymptotic structure of the receptivity problem. Region I: inviscid main layer (of $O(\delta)$ ), region II: viscous Stokes layer (of $O\left(R^{-1 / 2} \delta\right)$ ). The structure remains the same for the local streamwise velocity and temperature perturbations

it cause additional error because it is in fact the residue incurred in the $\mathrm{N}-\mathrm{S}$ equations when the Blasius solution is used as an approximation for the steady base flow. A buffer region is imposed as the outflow condition downstream so that the waves will not be reflected back into the computational domain. The Navier-Stokes Characteristic Boundary Condition (NSCBC) [77] is employed at the upper boundary. The fifth-order upwind and sixth-order central finite-difference schemes are employed to discretise the non-linear and viscous terms, respectively, and the second-order Runge-Kutta method is used for time advancing. More details can be found in [76].

\section{A large-Reynolds-number asymptotic theory}

Asymptotic analysis is now performed in order to probe into the detailed process of the excitation. This will provide a priori estimate for the amplitude of the mode generated by each type of the wall perturbations, and furthermore establish the quantitative relations between the three receptivity problems. Eventually, we will present an improved asymptotic theory, which gives quantitatively satisfactory predictions at moderate Reynolds numbers.

\subsection{Local receptivity mechanism}

The receptivity to a local wall perturbation appears rather simple: the time-periodic wall disturbance produces a forcing, which has a frequency and length scale comparable with those of the inviscid instability, and therefore excites an eigenmode.

At high Reynolds numbers, the unsteady disturbance in the main boundary layer is of inviscid nature, governed by the compressible Euler equations, which can be reduced to the compressible Rayleigh equation. Since the inviscid solution in the main layer does not satisfy the no-slip condition at the wall, a viscous Stokes layer in an $O\left(R^{-1 / 2}\right)$ vicinity of the wall needs to be introduced. For an eigenmode, the viscous effect from this layer provides an $O\left(R^{-1 / 2}\right)$ correction to the dispersion relation. The Stokes layer must be considered as well in order to account for the forcing by the wall perturbations. It follows that the excited flow field acquires a two-layered structure: the main layer I and the thin Stokes layer II adjacent to the wall, as is shown in Fig. 2.

In the case of blowing/suction, the boundary condition on the wall-normal velocity may directly be imposed on the inviscid flow in the main layer, unaffected by the sandwiching Stokes layer, as the analysis will show. However, the Stokes layer plays a substantial role in the cases of the forcing by the streamwise velocity and temperature perturbations because neither of these boundary conditions could possibly be imposed on the inviscid flow. Instead, each of these wall perturbations generates in the Stokes layer a viscous response, which has an $O\left(\epsilon_{w}\right)$ streamwise velocity or temperature. From the continuity equation it follows that the associated transverse velocity is $O\left(R^{-1 / 2} \epsilon_{w}\right)$, and remains of this size at the outer edge of the Stokes layer. The $O\left(R^{-1 / 2} \epsilon_{w}\right)$ unsteady outflux acts on the main layer to generate an $O\left(R^{-1 / 2} \epsilon_{w}\right)$ perturbation, from which an $O\left(R^{-1 / 2} \epsilon_{w}\right)$ discrete mode will emerge downstream.

The qualitative asymptotic argument leads to the a priori estimate for the magnitude of the inviscid mode excited by each type of the wall perturbations. 
5.2 Receptivity of the boundary-layer inviscid mode

In order to make quantitative predictions of the receptivity, we now analyse the viscous Stokes layer and its impact on the inviscid disturbance in the main layer. The ensuing analysis differs from and is simpler than that in [57] because of different and simpler forms of excitations.

\subsubsection{Stokes-layer solution}

The solution in this layer is described by a local coordinate

$$
Y=\left(\frac{R}{C_{w}}\right)^{1 / 2} y
$$

where $C_{w}=\mu_{w} T_{w}$ with $T_{w}$ and $\mu_{w}$ being the temperature and dynamic viscosity at the wall, respectively.

The perturbation in (3) can be expressed as

$$
\frac{1}{2} \epsilon_{w}\left[U_{p l},\left(\frac{R}{C_{w}}\right)^{-1 / 2} V_{p l}, W_{p l}, \rho_{p l}, \theta_{p l}, P_{p l}\right] \mathrm{e}^{\mathrm{i}(\beta z-\omega t)}+\text { c.c. }+\cdots,
$$

where $l=V, U, T$ distinguished three forms of wall perturbations. Substituting (19) along with (18) into the N-S equations, retaining the leading-order terms only and performing Fourier transform $\hat{\boldsymbol{\phi}}_{p l}(k, y)=\mathscr{F}\left[\boldsymbol{\phi}_{p l}(x, y)\right]$, we obtain the governing equations,

$$
\begin{aligned}
& -\mathrm{i} \omega T_{w} \hat{\rho}_{p l}+\mathrm{i} k \hat{U}_{p l}+\hat{V}_{p l, Y}+\mathrm{i} \beta \hat{W}_{p l}=0, \\
& -\mathrm{i} \omega\left(\hat{U}_{p l}, \hat{W}_{p l}\right)=-T_{w}\left(\mathrm{i} k \hat{P}_{p l}, \mathrm{i} \beta \hat{P}_{p l}\right)+\left(\hat{U}_{p l, Y Y}, \hat{W}_{p l, Y Y}\right), \\
& -\mathrm{i} \omega \hat{\theta}_{p l}=-\mathrm{i} \omega T_{w}(\gamma-1) M^{2} \hat{P}_{p l}+\frac{\hat{\theta}_{p l, Y Y}}{P r}, \\
& \hat{P}_{p l, Y}=0, \quad T_{w} \hat{\rho}_{p l}+\frac{\hat{\theta}_{p l}}{T_{w}}=\gamma M^{2} \hat{P}_{p l},
\end{aligned}
$$

where ()$_{Y} \equiv \partial / \partial Y$. The disturbance in the Stokes layer is compressible in that the density and velocity fluctuations are coupled through the continuity equation. For blowing/suction, the boundary condition at the wall is

$$
\left[\hat{U}_{p V}, \hat{V}_{p V}, \hat{W}_{p V}, \hat{\theta}_{p V}\right](k, 0)=\left[0,\left(\frac{R}{C_{w}}\right)^{1 / 2} \hat{F}(k), 0,0\right] \text { at } Y=0
$$

for streamwise velocity perturbation, the boundary condition at the wall is

$$
\left[\hat{U}_{p U}, \hat{V}_{p U}, \hat{W}_{p U}, \hat{\theta}_{p U}\right](k, 0)=[\hat{F}(k), 0,0,0] \text { at } Y=0
$$

for temperature perturbation, the boundary condition at the wall is

$$
\left[\hat{U}_{p T}, \hat{V}_{p T}, \hat{W}_{p T}, \hat{\theta}_{p T}\right](k, 0)=[0,0,0, \hat{F}(k)] \text { at } Y=0 \text {. }
$$

For wall blowing/suction, solving (20) subject to (21) we obtain

$$
\begin{aligned}
& \left(\hat{U}_{p V}, \hat{W}_{p V}\right)=(k, \beta) T_{w} \omega^{-1}\left(1-\mathrm{e}^{(-\mathrm{i} \omega)^{1 / 2} Y}\right) \hat{P}_{p V}, \\
& \hat{V}_{p V}=\left(R / C_{w}\right)^{1 / 2} \hat{F}(k)+\hat{P}_{p V}\left[\left(\mathrm{i} \omega M^{2}+\frac{\left(k^{2}+\beta^{2}\right) T_{w}}{\mathrm{i} \omega}\right) Y+G_{\mathrm{V}}\right], \\
& \hat{\theta}_{p V}=M^{2} T_{w}(\gamma-1)\left(1-\mathrm{e}^{(-\mathrm{i} \omega P r)^{1 / 2} Y}\right) \hat{P}_{p V}, \\
& \hat{\rho}_{p V}=\frac{M^{2}}{T_{w}}\left[1+(\gamma-1) \mathrm{e}^{(-\mathrm{i} \omega P r)^{1 / 2} Y}\right] \hat{P}_{p V},
\end{aligned}
$$


where we have put

$G_{\mathrm{V}}=-\frac{\mathrm{i}(\gamma-1) \omega M^{2}}{(-\mathrm{i} \omega P r)^{1 / 2}}\left[1-\mathrm{e}^{(-\mathrm{i} \omega P r)^{1 / 2} Y}\right]-\frac{\left(k^{2}+\beta^{2}\right) T_{w}}{(-\mathrm{i} \omega)^{3 / 2}}\left[1-\mathrm{e}^{(-\mathrm{i} \omega)^{1 / 2} Y}\right]$,

and $(-\mathrm{i})^{1 / 2} \equiv \exp (3 \pi \mathrm{i} / 4)$ such that $\exp \left\{(-\mathrm{i} \omega)^{1 / 2} Y\right\}$ attenuates exponentially with $Y$. Note that the first term in (24) represents the response to the suction and is asymptotically large. Formally, it should appear at a separate order, but for brevity of presentation it is tacitly included with the $O(1)$ remaining terms and $\hat{U}_{p V}, \hat{W}_{p V}$ and $\hat{\theta}_{p V}$, which represent the viscous correction to the eigenmode. It follows from (24b) that as $Y \rightarrow \infty$,

$\hat{V}_{p V} \rightarrow\left(R / C_{w}\right)^{1 / 2} \hat{F}(k)+\left[\left(\mathrm{i} \omega M^{2}+\frac{\left(k^{2}+\beta^{2}\right) T_{w}}{\mathrm{i} \omega}\right) Y+G_{V, \infty}\right] \hat{P}_{p V}$,

with

$G_{V, \infty}=-\frac{\mathrm{i}(\gamma-1) \omega M^{2}}{(-\mathrm{i} \omega \operatorname{Pr})^{1 / 2}}-\frac{\left(k^{2}+\beta^{2}\right) T_{w}}{(-\mathrm{i} \omega)^{3 / 2}}$.

On right-hand side of (25), the term proportional to $Y$ matches automatically the impermeability behaviour of the main-layer solution, while the $Y$-independent terms represent the transpiration velocity, or outflux, induced by the viscous motion in the Stokes layer. Of the two terms, the second corresponds to the viscous correction to the dispersion of the eigenmode, while the first is the equivalent blowing/suction velocity to drive the inviscid disturbance in the main layer, and is the only term required if the solution for the receptivity is to be obtained to leading-order accuracy. As is expected, the blowing/suction effect is transmitted across the Stokes layer without being hindered.

In the case of streamwise velocity perturbation, $\hat{U}_{p U}$ and $\hat{V}_{p U}$ are replaced, respectively, by

$\hat{U}_{p U}=k T_{w} \omega^{-1}\left(1-\mathrm{e}^{(-\mathrm{i} \omega)^{1 / 2} Y}\right) \hat{P}_{p U}+\hat{F} \mathrm{e}^{(-\mathrm{i} \omega)^{1 / 2} Y}$,

$\hat{V}_{p U}=\frac{\mathrm{i} k \hat{F}}{(-\mathrm{i} \omega)^{1 / 2}}\left[1-\mathrm{e}^{(-\mathrm{i} \omega)^{1 / 2} Y}\right]+\left[\left(\mathrm{i} \omega M^{2}+\frac{\left(k^{2}+\beta^{2}\right) T_{w}}{\mathrm{i} \omega}\right) Y+G_{V}\right] \hat{P}_{p U}$.

As $Y \rightarrow \infty$,

$\hat{V}_{p U} \rightarrow \frac{\mathrm{i} k \hat{F}}{(-\mathrm{i} \omega)^{1 / 2}}+\left[\left(\mathrm{i} \omega M^{2}+\frac{\left(k^{2}+\beta^{2}\right) T_{w}}{\mathrm{i} \omega}\right) Y+G_{V, \infty}\right] \hat{P}_{p U}$.

For temperature perturbation, $\hat{\theta}_{p T}, \hat{\rho}_{p T}$ and $\hat{V}_{p T}$ are found as

$\hat{\theta}_{p T}=\hat{F} \mathrm{e}^{(-\mathrm{i} \omega P r)^{1 / 2} Y}+M^{2} T_{w}(\gamma-1)\left(1-\mathrm{e}^{(-\mathrm{i} \omega P r)^{1 / 2} Y}\right) \hat{P}_{p T}$,
$\hat{\rho}_{p T}=-\hat{F} \mathrm{e}^{(-\mathrm{i} \omega P r)^{1 / 2} Y} / T_{w}^{2}+\frac{M^{2}}{T_{w}}\left[1+(\gamma-1) \mathrm{e}^{(-\mathrm{i} \omega P r)^{1 / 2} Y}\right] \hat{P}_{p T}$,
$\hat{V}_{p T}=\frac{\mathrm{i} \omega \hat{F}}{T_{w}(-\mathrm{i} \omega P r)^{1 / 2}}\left[1-\mathrm{e}^{(-\mathrm{i} \omega P r)^{1 / 2} Y}\right]+\left[\left(\mathrm{i} \omega M^{2}+\frac{\left(k^{2}+\beta^{2}\right) T_{w}}{\mathrm{i} \omega}\right) Y+G_{V}\right] \hat{P}_{p T}$.

As $Y \rightarrow \infty$,

$\hat{V}_{p T} \rightarrow \frac{\mathrm{i} \omega \hat{F}}{T_{w}(-\mathrm{i} \omega P r)^{1 / 2}}+\left[\left(\mathrm{i} \omega M^{2}+\frac{\left(k^{2}+\beta^{2}\right) T_{w}}{\mathrm{i} \omega}\right) Y+G_{V, \infty}\right] \hat{P}_{p T}$.

\subsubsection{Main-layer solution}

The disturbance in the main layer is driven by the transpiration velocity at the outer edge of the Stokes layer. Its solution is expressed as

$\tilde{\varphi}_{p}(x, y, z, t)=\frac{1}{2} \epsilon_{w}\left(\varphi_{p}(x, y)+O\left(R^{-1 / 2}\right)\right) \mathrm{e}^{\mathrm{i}(\beta z-\omega t)}+$ c.c., 
where $\varphi=[v, p]^{\mathrm{T}}$.

Substitution of (30) into the $\mathrm{N}-\mathrm{S}$ equations leads to the linearised Euler equations, which reduce, after performing Fourier transform with respect to $x$, to the homogeneous Rayleigh equations,

$\boldsymbol{L}_{R}\left(d_{y} ; \omega, k, \beta\right) \hat{\boldsymbol{\varphi}}_{p}(k, y)=0$,

where $\hat{\boldsymbol{\varphi}}_{p}(k, y)=\mathscr{F}\left[\boldsymbol{\varphi}_{p}(x, y)\right], \boldsymbol{L}_{R}$ is the Rayleigh operator,

$\boldsymbol{L}_{R}\left(d_{y} ; \omega, k, \beta\right) \equiv \boldsymbol{I} \frac{\mathrm{d}}{\mathrm{d} y}-\left(\begin{array}{cc}\mathrm{i} k U_{B}^{\prime} / S_{0}-M^{2} S_{0}-T_{B}\left(k^{2}+\beta^{2}\right) / S_{0} \\ -S_{0} / T_{B}\end{array}\right)$,

with $S_{0}=\mathrm{i}\left(k U_{B}-\omega\right)$. The attenuation condition,

$\hat{p}_{p}(k, y) \rightarrow 0$ as $y \rightarrow \infty$,

is imposed in the far field.

As (25), (27) and (29) indicate, each form of the wall perturbations generates an equivalent wall-normal velocity acting the bottom of the main deck. It is convenient to consider a unit forcing by specifying the lower boundary condition for (31) as

$\hat{v}_{p}(k, 0)=1$.

Once the boundary-value problem of (31) with (32) and (33) is solved (as described below) to obtain $\hat{v}_{p}$ and $\hat{p}_{p}$, the corresponding streamwise velocity component $\hat{u}_{p}$ is obtained from the $x$-momentum equation. The solution for each type of wall perturbation follows immediately by multiplying $\hat{v}_{p}$ and $\hat{u}_{p}$ by the appropriate factor according to (25), (27) and (29), respectively.

\subsubsection{Receptivity coefficient}

As in the finite- $R$ approach in Sect. 3, the solution for $\varphi_{p}$ in physical space is obtained by performing inverse Fourier transform,

$\boldsymbol{\varphi}_{p}(x, y) \equiv \mathscr{F}^{-1}\left[\hat{\boldsymbol{\varphi}}_{p}(k, y)\right]=\frac{1}{\sqrt{2 \pi}} \int_{-\infty}^{\infty} \hat{\boldsymbol{\varphi}}_{p}(k, y) \mathrm{e}^{\mathrm{i} k x} \mathrm{~d} k$.

The integrand has poles at $k=\alpha$, where $\alpha$ is the complex streamwise wavenumber of the eigenmode of (31) subject to the impermeability condition (or the impedance condition in the improved asymptotic theory to be discussed later). Using the residue theorem, we obtain the asymptotic perturbation in the downstream limit,

$\boldsymbol{\varphi}_{p}(x, y) \rightarrow \frac{\sqrt{2 \pi} \mathrm{i}}{\left[\partial\left(\hat{\boldsymbol{\varphi}}_{p}\right)^{-1} / \partial k\right]_{k=\alpha}} \mathrm{e}^{\mathrm{i} \alpha x}$ as $x \rightarrow \infty$,

where the pre-factor of $\mathrm{e}^{\mathrm{i} \alpha x}$ represents the equivalent amplitude at the wall-perturbation centre. Here $\left[\partial\left(\hat{\boldsymbol{\varphi}}_{p}\right)^{-1} / \partial k\right]_{k=\alpha}$ is calculated following the similar procedure in the finite- $R$ approach as described in Sect. 3.

For the blowing/suction, the streamwise velocity $\hat{u}_{p V}$ is given by $\hat{u}_{p}$ multiplied by the factor $\left(R / C_{w}\right)^{1 / 2} \hat{F}(\alpha)$ according to (25), as was remarked earlier. The initial amplitude of the excited mode, normalised by wall-perturbation intensity $\epsilon_{w}$, can be expressed as

$\mathscr{A}_{V}=\left(\frac{R}{C_{w}}\right)^{1 / 2} \hat{F}(\alpha) \lim _{x \rightarrow \infty} \max _{y} u_{p}(x, y) \mathrm{e}^{-\mathrm{i} \alpha x}$.

The receptivity coefficient $\Lambda_{V}$, defined as the asymptotic amplitude of the generated instability mode to that of the wall perturbation, is

$\Lambda_{V}=\mathscr{A}_{V} / \hat{F}(\alpha)$. 
Since each of three wall perturbations generates an equivalent forcing in the form of an unsteady transverse velocity (outflux) acting at the bottom of the main layer, there exist close connections between the receptivity in the three cases. Let the receptivity coefficients for the streamwise and temperature perturbation be denoted by $\Lambda_{U}$ and $\Lambda_{T}$, respectively. Using the relations with the solution subject to the unit forcing (33), we can deduce, according to (25), (27) and (29), that the ratios $\Lambda_{U} / \Lambda_{V}$ and $\Lambda_{T} / \Lambda_{V}$ have simple expressions,

$\frac{\Lambda_{U}}{\Lambda_{V}}=\frac{\left(R / C_{w}\right)^{-1 / 2} \mathrm{i} \alpha}{(-\mathrm{i} \omega)^{1 / 2}}, \quad \frac{\Lambda_{T}}{\Lambda_{V}}=\frac{\left(R / C_{w}\right)^{-1 / 2} \mathrm{i} \omega}{T_{w}(-\mathrm{i} \omega \operatorname{Pr})^{1 / 2}}$.

Obviously, the same ratios hold for the initial amplitude,

$\mathscr{A}_{U}=\frac{\left(R / C_{w}\right)^{-1 / 2} \mathrm{i} \alpha}{(-\mathrm{i} \omega)^{1 / 2}} \mathscr{A}_{V}, \quad \mathscr{A}_{T}=\frac{\left(R / C_{w}\right)^{-1 / 2} \mathrm{i} \omega}{T_{w}(-\mathrm{i} \omega P r)^{1 / 2}} \mathscr{A}_{V}$.

These relations are of practical use because once the receptivity property to blowing/suction is known, that to the streamwise velocity or temperature perturbations follows immediately.

\subsection{Improved large-Reynolds-number theories for instability and receptivity}

For an instability mode, the leading-order perturbation in the main layer satisfies the Rayleigh equations,

$L_{R} \hat{\varphi}_{I}=0$,

subject to the impermeability condition, $\hat{v}_{I}(0)=0$, and the far-field attenuation condition that $\hat{p}_{I} \rightarrow 0$ as $y \rightarrow \infty$. The error due to neglecting the viscous diffusion terms in the main-layer equations is merely of $O\left(R^{-1}\right)$. However, neglecting the viscous effect in the Stokes layer causes a much larger $O\left(R^{-1 / 2}\right)$ error to the dispersion relation. For the same reason, an error of $O\left(R^{-1 / 2}\right)$ is present in the purely inviscid receptivity theory presented above. As a result, the solutions for instability and receptivity are not expected to be as accurate as their finite- $R$ counterparts. In order to improve the accuracy of the large- $R$ asymptotic instability and receptivity theories for moderate Reynolds numbers, we may now propose theories by taking into account the $O\left(R^{-1 / 2}\right)$ viscous correction induced by the Stokes layer, which is required in order to satisfy the no-slip boundary condition. In the improved approach, the instability mode remains being governed by the Rayleigh equation (40), but the Stokes-layer correction is taken into account simply by replacing the impermeability condition by

$\hat{v}_{I}(0)=-\left(\frac{R}{C_{w}}\right)^{-1 / 2}\left[\frac{\mathrm{i}(\gamma-1) \omega M^{2}}{(-\mathrm{i} \omega \operatorname{Pr})^{1 / 2}}+\frac{\left(\alpha^{2}+\beta^{2}\right) T_{w}}{(-\mathrm{i} \omega)^{3 / 2}}\right] \hat{p}_{I}(0)$,

where the pre-factor of $\hat{p}_{I}$ plays the role of 'impedance'. By imposing the 'impedance boundary condition' (41), the error is reduced to $O\left(R^{-1}\right)$, and the accuracy of the dispersion relation at moderate Reynolds numbers is improved remarkably as will be shown below.

Similarly, for receptivity calculations the wall boundary condition (33) is replaced by

$\hat{v}_{p}(k, 0)=1-\left(\frac{R}{C_{w}}\right)^{-1 / 2}\left[\frac{\mathrm{i}(\gamma-1) \omega M^{2}}{(-\mathrm{i} \omega P r)^{1 / 2}}+\frac{\left(k^{2}+\beta^{2}\right) T_{w}}{(-\mathrm{i} \omega)^{3 / 2}}\right] \hat{P}_{p}$.

\section{Numerical results}

6.1 The base flow and linear stability characteristics of inviscid modes

This paper considers mainly two different oncoming conditions as shown in Table 1 . The parameters in conditions 1 and 2 are chosen to be the same as those in [50] and [78,79], respectively, but a few different temperatures are also considered in each case. Sutherland's viscosity is used for all the calculations. The streamwise velocities $U_{\mathrm{B}}$ and 
Table 1 Parameters characterising the base flow

\begin{tabular}{lllllllll}
\hline Condition & $M$ & $T_{\infty}(K)$ & $R$ & References & $T_{w}$ & $\delta_{99}$ & $y_{c}$ & $U_{c}$ \\
\hline 1 & 4.5 & 65.15 & 1000 & {$[50]$} & 4.4 & 13.5 & 11.1 & 0.89 \\
2 & 5.92 & 48.69 & 1560 & {$[78,79]$} & 6.9 & 20.0 & 18.2 & 0.94 \\
\hline
\end{tabular}

(a)

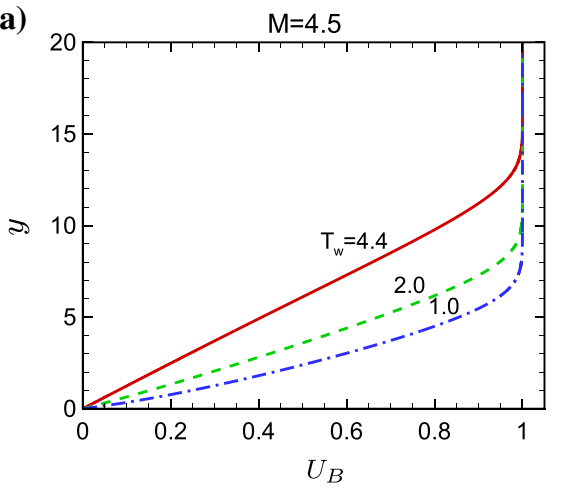

(b)

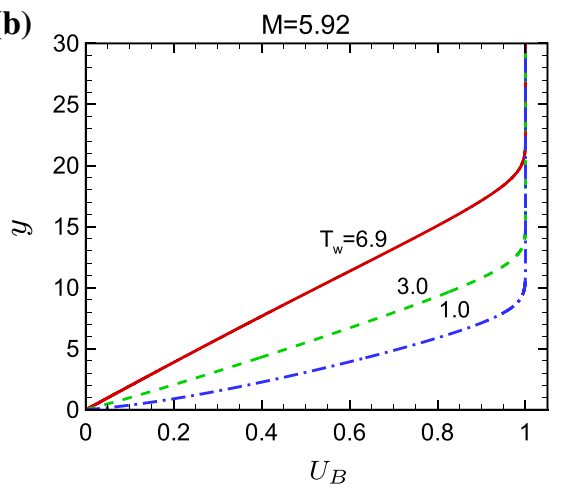

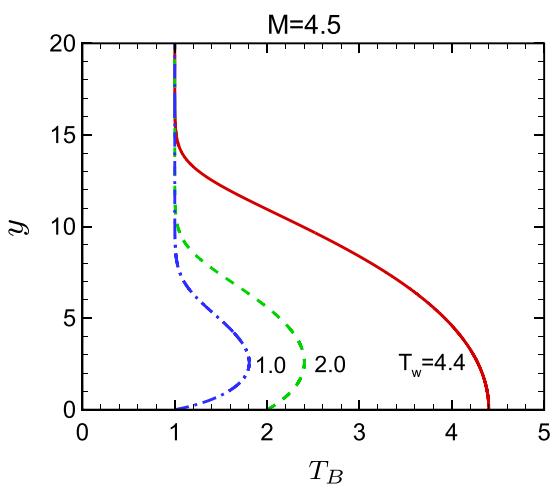

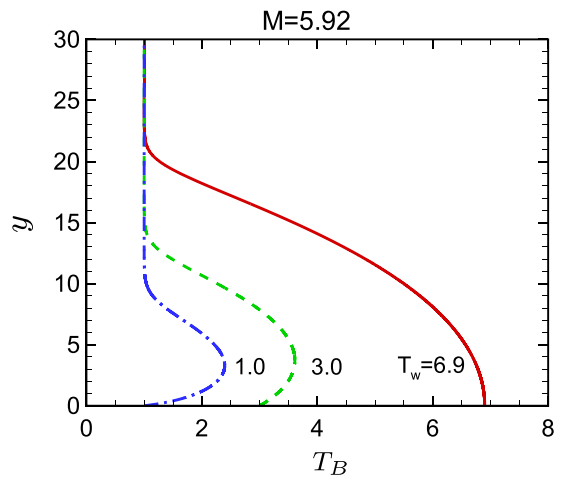

Fig. 3 The streamwise velocity and temperature profiles of the Blasius boundary layer under conditions 1 (a) and 2 (b) with different wall temperatures

temperatures $T_{\mathrm{B}}$ for the two conditions are shown in Fig. 3. The nominal boundary-layer thicknesses $\delta_{99}$, the wall temperatures $T_{w}$ are displayed in Table 1. Figure 3 also displays the result for other wall temperatures. Wall cooling reduces the boundary-layer thickness and changes the characteristics of the temperature and velocity profiles. For $T_{w}=4.4(M=4.5)$ and $T_{w}=6.9(M=5.92)$, the maximum temperature occurs on the wall and decreases monotonically away from it. However, for cooled wall cases (e.g. $T_{w}=4.4$ for $M=4.5$ and $T_{w}=6.9$ for $M=5.92$ ), the temperature increases first with the distance from the wall, reaches its maximum in the boundary layer and then decreases.

Figure 4 shows the dependence of the phase speed and growth rate on the frequency predicted by linear stability theory (i.e. O-S approach) and Rayleigh equation for the two conditions with different wall temperatures. In each case, first modes are in the lower frequency range, while second modes are in the higher frequency range, with the former being in the range where $c_{r}$ increases with $\omega$ monotonically until reaching $U_{c}$, the phase speed of the upper-branch neutral first mode. As $\omega \rightarrow 0$, the phase speed of first modes approaches $1-1 / M$, the phase speed of the slow acoustic wave at zero incident angle. At both conditions, second modes are more unstable overall. Although not shown, the second modes can be continued towards the lower-frequency limit, in which they are referred to $\mathrm{F}$ modes because their phase speeds approach $c=1+1 / M$, the phase speed of the fast acoustic wave [39]. A band of 

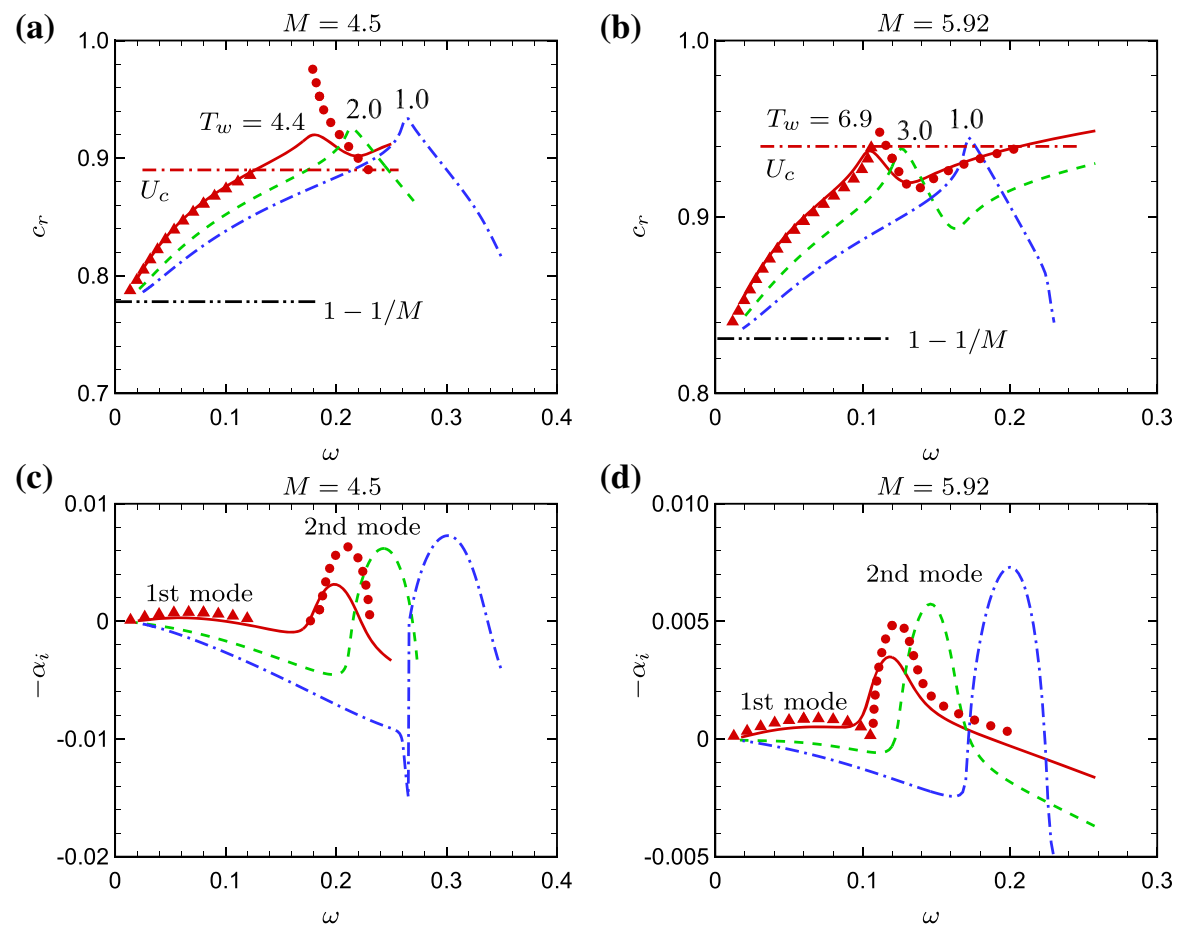

Fig. 4 Dependence on the frequency $\omega$ of the phase speed $c_{r}$ and the growth rate $-\alpha_{i}$ of 2D modes $(\beta=0)$ under condition 1 (a, c) and condition $2(\mathbf{b}, \mathbf{d})$ with different wall temperatures. Red/green/blue lines: the O-S solutions; triangles/circles: first/second mode obtained by solving the Rayleigh equation

unstable first modes exists for the adiabatic wall temperature, but when the wall is cooled sufficiently, the unstable band of first modes disappears. As is well known, wall cooling has a strong stabilisation effect on the first mode while destabilising the second mode.

For condition 2, the first- and second-mode brands are no longer separated. As a result, the phase speeds of the first and second modes become equal at a particular frequency (Fig. 4b), which is referred to as the synchronisation point, despite the fact that their growth rates differ by a finite amount that is numerically small [39].

In Fig. 5, the phase speed and growth rate obtained by three approaches are compared. The purely inviscid Rayleigh theory captures the qualitative instability characteristics, but appreciable quantitative difference exists. The accuracy is improved considerably by including the viscous Stokes-layer correction. Figure 6 compares the phase speeds and growth rates obtained by the O-S, the Rayleigh and the improved Rayleigh approaches for three representational frequencies in the $2 \mathrm{D}$ second-mode band under conditions 1 and 2 . All three give the same result for sufficiently large $R$. The improved Rayleigh approach increases the accuracy significantly, and satisfactory agreement with the $\mathrm{O}-\mathrm{S}$ prediction is observed even for $R$ of a few thousands.

The profiles of the $\widehat{p}$ and $\widehat{u}$ components of the eigenfunction under conditions 1 and 2 are displayed in Fig. 7 for instability modes with $(M, \omega)=(4.5,0.19)$ (left column) and $(M, \omega)=(5.92,0.11)$ (right column), where all the quantities are normalised by the amplitude of the wall pressure. The overall shape of the pressure eigenfunction across the entire boundary condition is well predicted by the three approaches with the improved Rayleigh equation giving a more accurate result closer to the $\mathrm{O}-\mathrm{S}$ prediction. The Rayleigh and the improved Rayleigh approaches give the correct distribution of the streamwise velocity only in the main layer. Fortunately, a composite solution for $\widehat{u}$, uniformly valid in the entire layer, can be constructed using the Stokes-layer solution for the streamwise velocity; see appendix for the detail. Figure 7(b) shows the composite solutions for the Rayleigh and improved Rayleigh approaches, of which the latter again gives an appreciably more accurate prediction. 
(a)

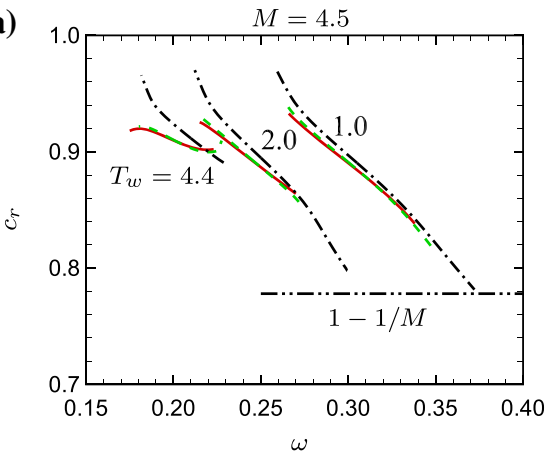

(b)

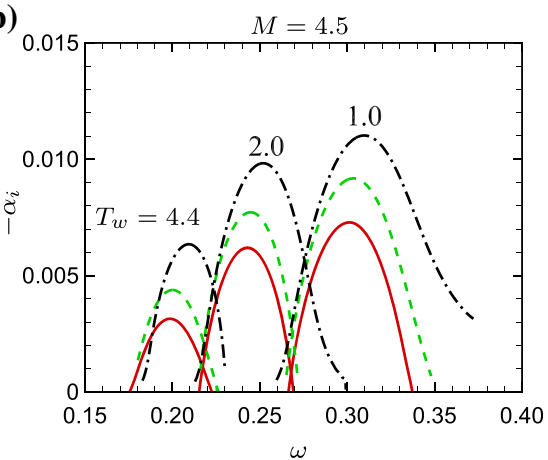

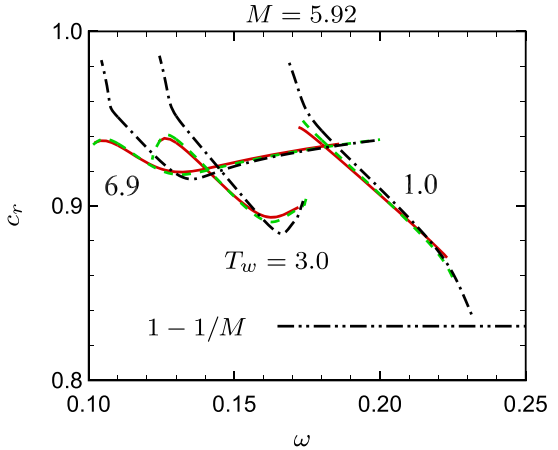

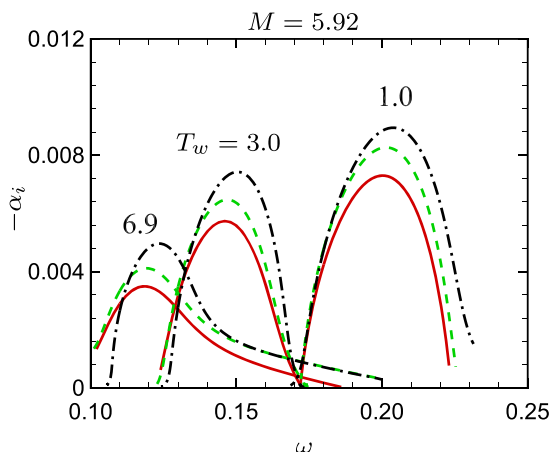

Fig. 5 Comparison of $c_{r}$ (a) and $-\alpha_{i}$ (b) obtained by the O-S (red solid lines), improved Rayleigh (green dashed lines) and Rayleigh (black dot-dashed lines) equations under condition 1 and 2 with different wall temperatures

(a)

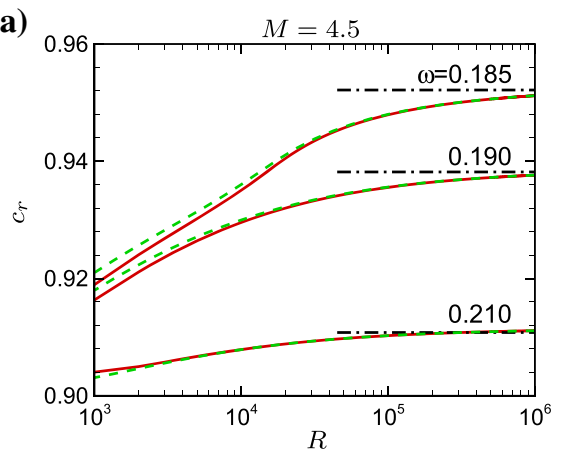

(b)

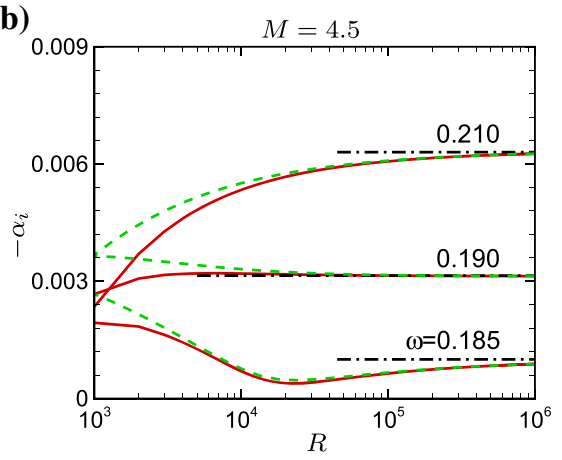

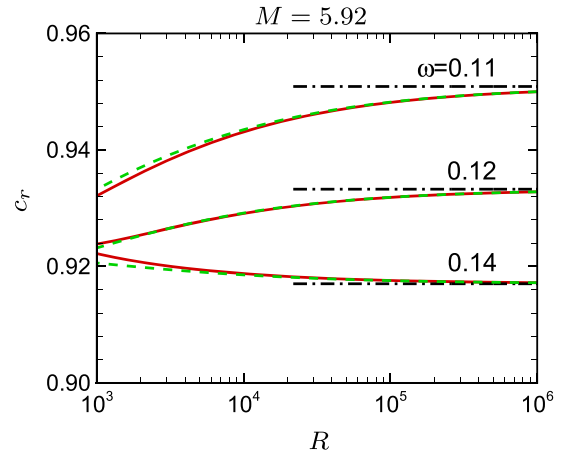

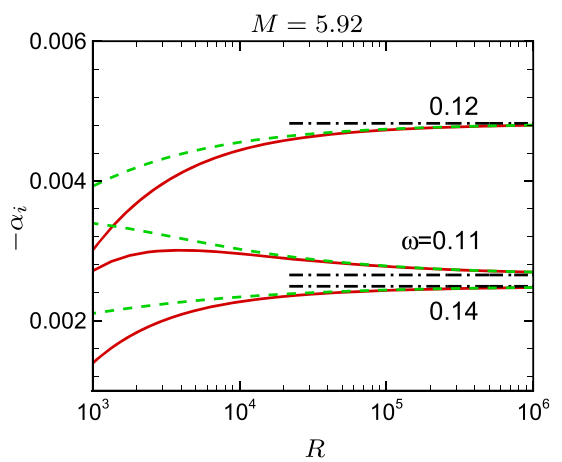

Fig. 6 Comparison of $c_{r}$ (a) and $-\alpha_{i}$ (b) obtained by the O-S (red solid lines), Rayleigh (black dot-dashed lines) and improved Rayleigh (green dashed lines) approaches under conditions 1 and 2 in a range of the Reynolds numbers. (Color figure online) 
(a)

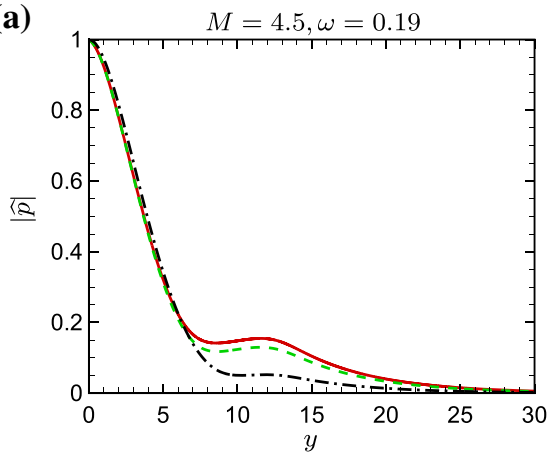

(b)

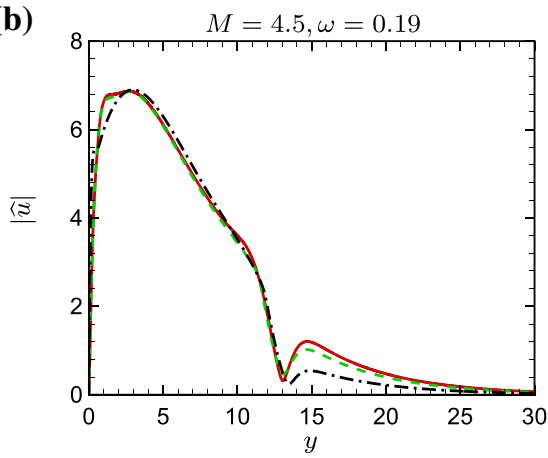

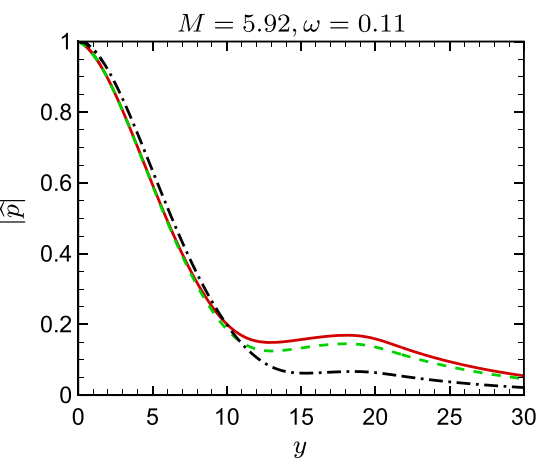

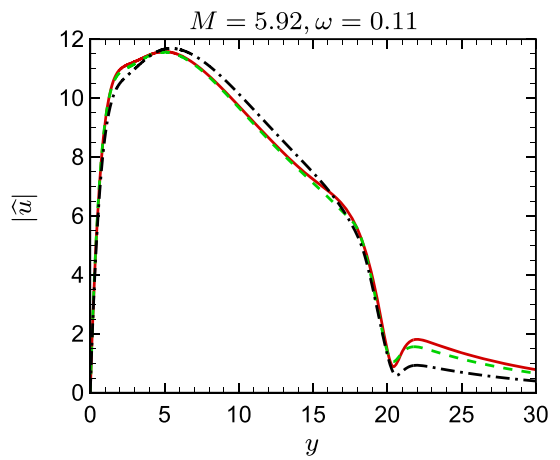

Fig. 7 Eigenfunctions $|\widehat{p}|$ (a) and $|\widehat{u}|$ (b) of the second mode obtained by the O-S (red solid lines), Rayleigh (black dot-dashed lines) and improved Rayleigh (green dashed lines) approaches under conditions 1 and 2. (Color figure online)
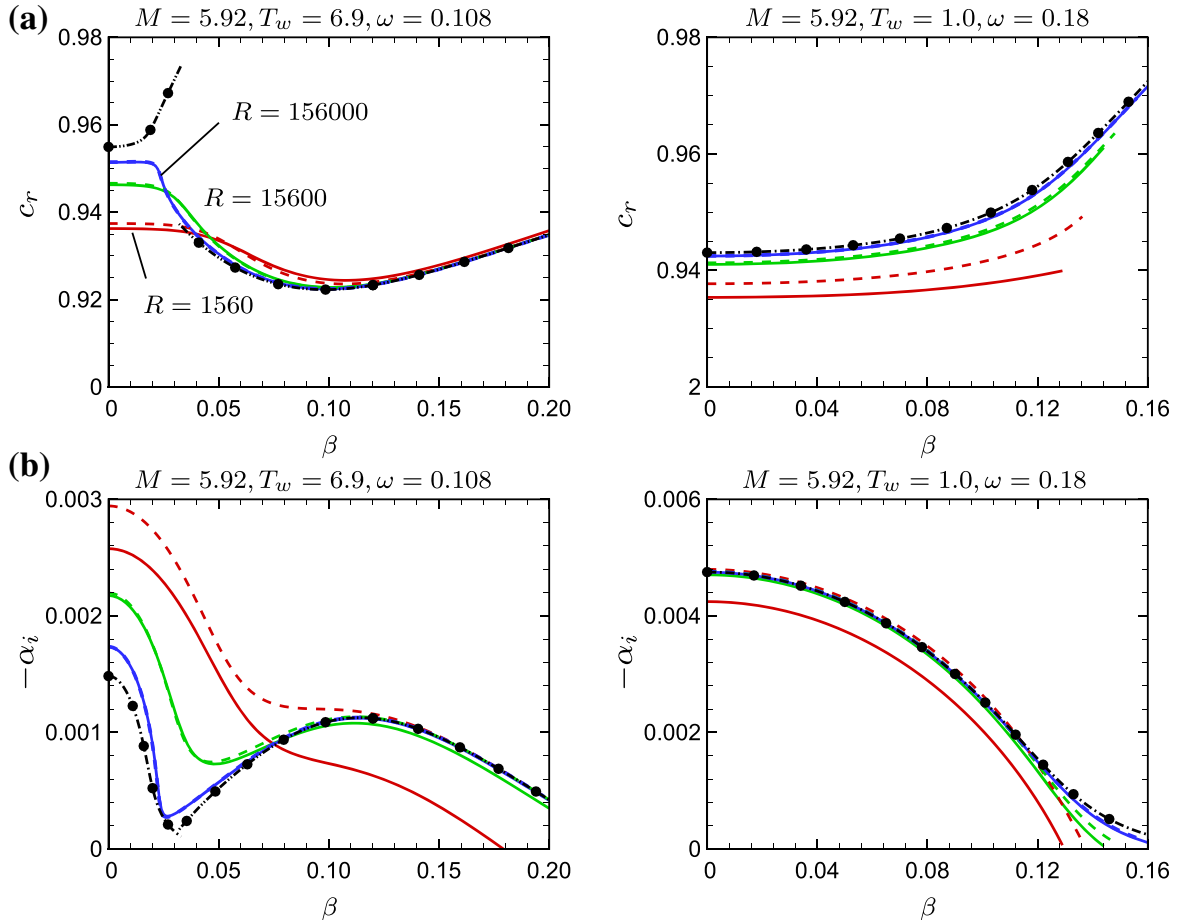

Fig. 8 Variation with $\beta$ of $c_{r}$ (a) and $-\alpha_{i}$ (b) obtained by the O-S (solid lines), improved Rayleigh (dashed lines) and Rayleigh (dot-dashed black lines with filled circles) under condition 2. The red, green and blue lines stand for the cases of $R=1560,15,600$ and 156,000 , respectively. (Color figure online) 

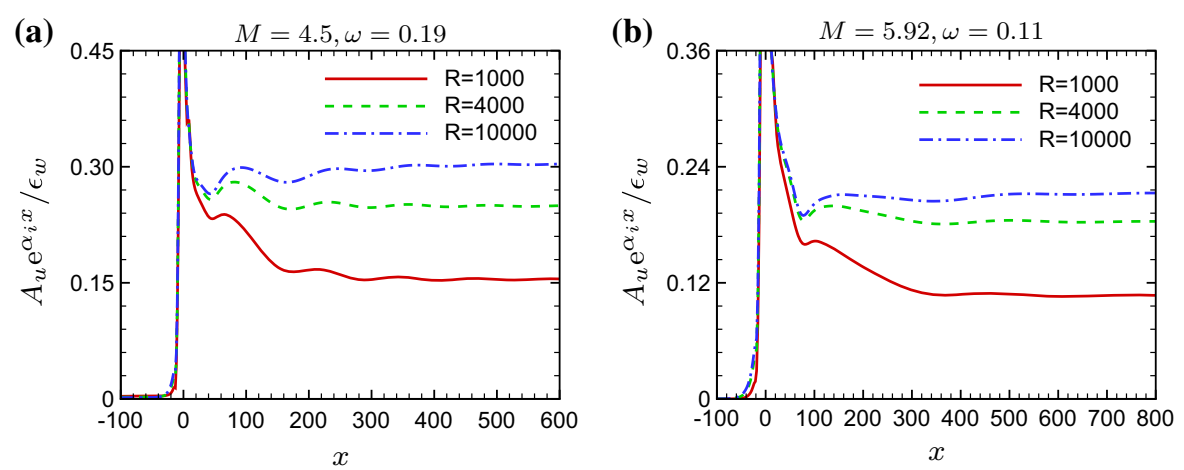

Fig. 9 The streamwise evolution of the streamwise velocity amplitude of the perturbation generated by wall blowing/suction for conditions 1 (a) and 2 (b)

Figure 8 displays the variation with $\beta$ of the phase speeds $c_{r}$ and growth rates $-\alpha_{i}$ of 3D instability modes under condition 2. For $T_{w}=6.9, \omega$ is chosen to be somewhat higher than the $2 \mathrm{D}$ synchronisation frequency. Results given by the Rayleigh equation exhibit two branches of normal modes, corresponding to the low- $\beta$ second mode and high- $\beta$ first mode. The phase speed of the former is higher and increases with $\beta$, whereas the phase speed of the latter is lower and decreases with $\beta$ before increasing. Although the phase speeds of the two modes do not intersect, they stay rather close to each other for $\beta \leq 0.02$. The growth rates of the two modes at $\beta \approx 0.02$ are also close, indicating that $(\omega, \beta) \approx(0.108,0.02)$ is near a synchronisation that is an extension of the point $(\omega, \beta) \approx(0.106,0)$ in Fig. 4 to a 3D configuration. In fact, the synchronisation points form a continuous curve starting from $(0.106,0)$ in the $\omega-\beta$ plane; for a small $\beta$, the synchronisation point is found at a frequency slightly higher than 0.106 . As $\beta$ increases further, the phase speeds of the two modes deviate from each other. The results given by the $\mathrm{O}-\mathrm{S}$ equation agree with those by the Rayleigh equation when $R$ is sufficiently large, but appreciable discrepancy appears when $R$ is moderate. The improved Rayleigh approach is able to predict both the phase speed and growth rate accurately when $R=O\left(10^{4}\right)$, and even for $R=O\left(10^{3}\right)$, there is a broad agreement between the $\mathrm{O}-\mathrm{S}$ and improved Rayleigh approaches. For $T_{w}=1.0$, the results correspond to second mode. The phase speed of the instability mode increases with $\beta$, whereas the growth rate decreases with $\beta . R$ has only a slightly influence on instability. The results given by the $\mathrm{O}-\mathrm{S}$ equation agree with those by the Rayleigh equation when $R$ is moderate (e.g. $R=O\left(10^{3}\right)$ ).

\subsection{The receptivity of inviscid modes}

\subsubsection{The receptivity to blowing/suction}

DNS were performed for the case of two-dimensional blowing/suction, for which the wall boundary condition is

$$
(u, v, T)=\left(0,-\epsilon_{w} \mathrm{e}^{-x^{2} / d^{2}} \cos (\omega t), T_{w}\right),
$$

where $\epsilon_{w}=10^{-6}$, for the base-flow conditions 1 and 2, we take $d=6.30$ and 9.32, respectively. In each case, the value of $d$ corresponds to $\Delta_{w} / \delta_{99}=2.0$, where $\Delta_{w}$ denotes the effective width of the Gaussian distribution, taken to be the interval where its value is over 0.01 of its maximum, and so $\Delta_{w}=4.292 d$. The parameter values pertain to conditions 1 and 2 .

The streamwise velocity of the excited perturbation is denoted as $\tilde{u}$. In order to illustrate the receptivity process quantitatively, in Fig. 9 we plot the amplitude of $\tilde{u}$,

$A_{u}(x)=\max _{y, t} \tilde{u}(x, y, t)$.

The excited perturbation consists of not only the unstable discrete mode, but also stable discrete modes as well as the near-field response. However, since the latter two in the downstream limit are overwhelmed by the exponentially 

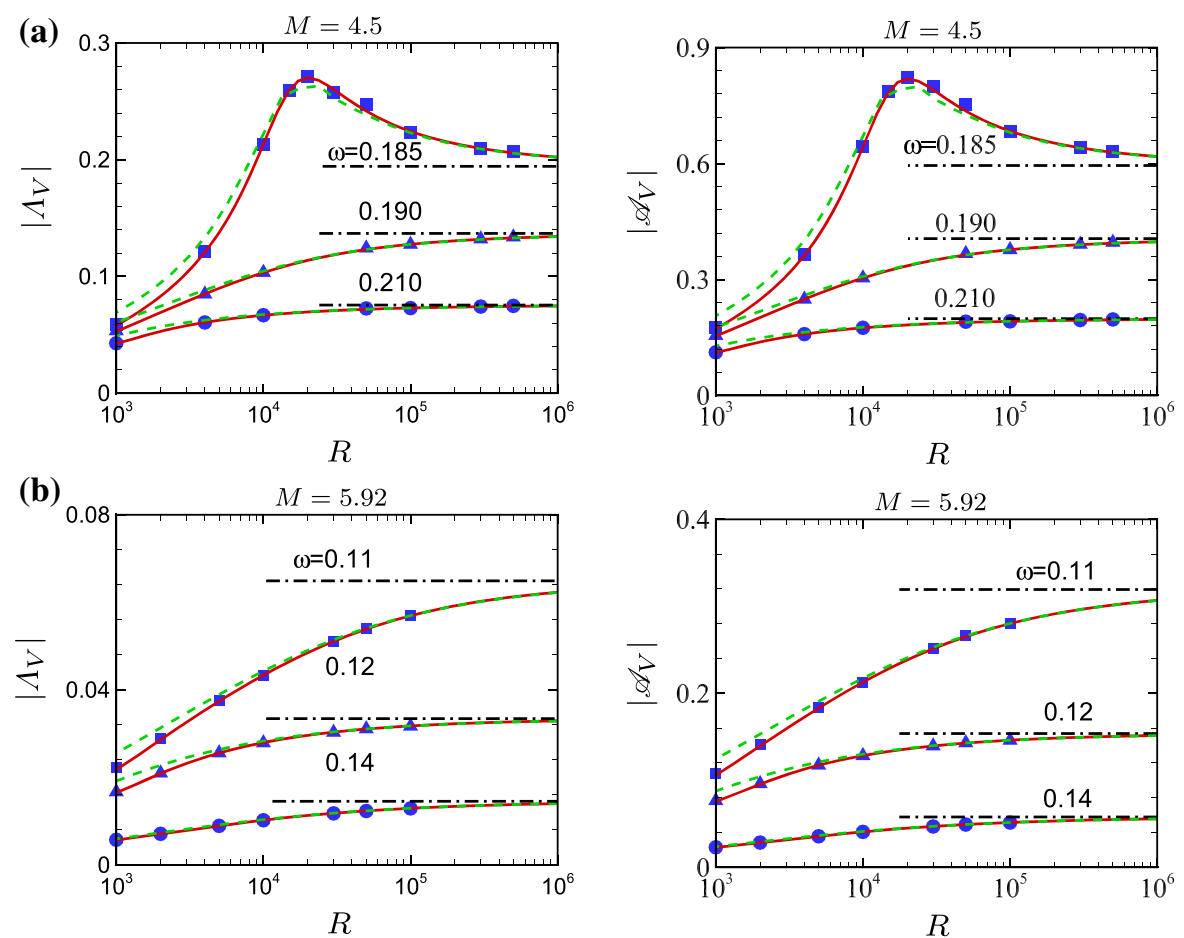

Fig. 10 The dependence of the receptivity coefficient $\left|\Lambda_{V}\right|$ and initial amplitude $\left|\mathscr{A}_{V}\right|$ on $R$ for blowing/suction under conditions 1 (a) and 2 (b), where the solid (red) lines, dot-dashed (black) lines, dashed (green) lines and symbols (blue) represent the results predicted by the finite- $R$, large- $R$ theory, improved large- $R$ theory and DNS, respectively. (Color figure online)

growing mode, $A_{u}$ can be regarded as the amplitude of the unstable discrete mode provided $x$ is sufficiently large. Since $A_{u}$ grows exponentially, we plot $A_{u} \exp \left(\alpha_{i} x\right)$ normalised by $\epsilon_{w}$. For all $R$ and $\omega, A_{u} \exp \left(\alpha_{i} x\right) / \epsilon_{w}$ approaches zero in the upstream limit, followed by an overshoot and finally approaches a constant in the downstream limit. The constant, representing the receptivity coefficient, increases with $R$.

The three theoretical approaches are implemented for the two conditions given in Table 1 . The results are displayed in Fig. 10. For each condition, three representational frequencies within the unstable frequency band of the second-mode instability are selected. As $R \rightarrow \infty$, the receptivity coefficient $\left|\Lambda_{V}\right|$ and initial amplitude $\left|\mathscr{A}_{V}\right|$ (red solid lines) approach the large- $R$ theory, shown as the black dot-dashed lines. The increase of $\left|\Lambda_{V}\right|$ and $\left|\mathscr{A}_{V}\right|$ with $R$ is monotonic except for $\omega=0.185$ in panel (a). The receptivity coefficient for $R=10^{3}$ could be only a third of that predicted by the simple inviscid theory, implying a considerable impact of the Stokes layer on the receptivity. Interestingly, although the Stokes-layer correction in the improved approach is only of $O\left(R^{-1 / 2}\right)$, taking this small correction into account leads to a much more accurate prediction of the receptivity. This is because the dispersion relation at a finite Reynolds number is more accurately predicted by the improved approach, as was shown in Fig. 5, and it is thus expected that the improved accuracy is expected for the two other forms of wall perturbations. The improved large- $R$ approach captures rather well the dependence on $R$ including remarkably the non-monotonic feature, and the agreement between the finite- $R$ and improved large- $R$ approaches is quite satisfactory even when $R$ is fairly moderate, e.g. $R=10^{3}$. In order to assess the accuracy of the theoretical predictions, the DNS results are presented in Fig. 10, and the agreement is excellent. However, it only takes less than just one second to perform one theoretical calculation, which is of course superior to DNS that requires one to two days.

Figure 11a displays the dependence on $\omega$ of the receptivity coefficient $\left|\Lambda_{V}\right|$ and initial amplitude $\left|\mathscr{A}_{V}\right|$ of the second mode under condition 1 . Strong receptivity occurs at both the lower- and upper-branch neutral frequencies, $\omega \approx 0.185$ and 0.23 . The strong receptivity at the upper branch is unimportant because the excited mode soon enters the decaying phase downstream. Now turn to the flow condition 2. Figure $11 \mathrm{~b}$ shows the dependence of $\left|\Lambda_{V}\right|$ and 

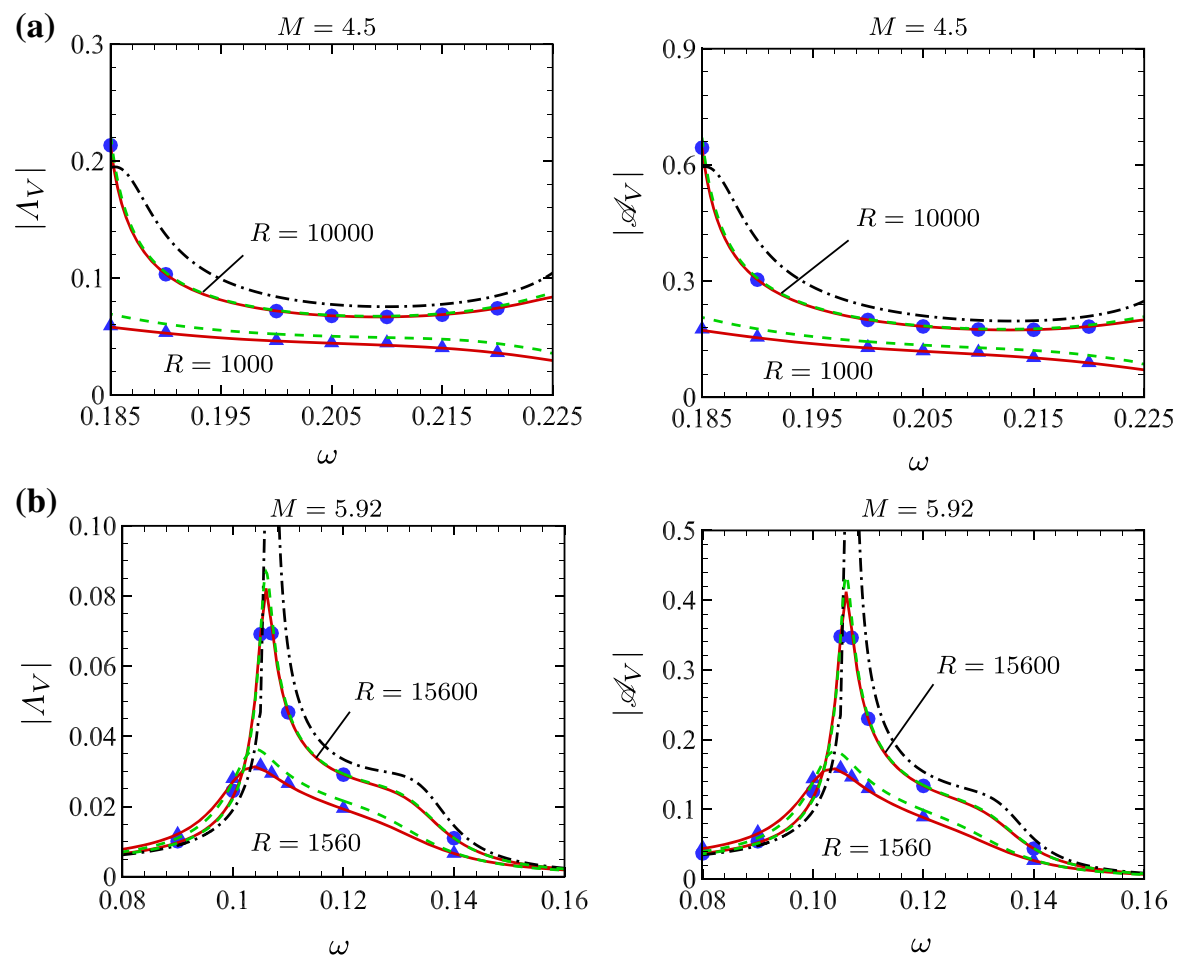

Fig. 11 The dependence of the receptivity coefficient $\left|\Lambda_{V}\right|$ and initial amplitude $\left|\mathscr{A}_{V}\right|$ on $\omega$ for blowing/suction under conditions 1 (a) and 2 (b), where the line types are the same as those in Fig. 10

$\left|\mathscr{K}_{V}\right|$ on $\omega$. Both $\left|\Lambda_{V}\right|$ and $\left|\mathscr{K}_{V}\right|$ exhibit sharp peaks at the synchronisation frequency, $\omega \approx 0.106$. As $\omega$ is reduced to be within the first-mode band, $\left|\Lambda_{V}\right|$ and $\left|\mathscr{K}_{V}\right|$ diminish rapidly. In the frequency window of the second-mode instability, $0.115<\omega<0.135,\left|\Lambda_{V}\right|$ and $\left|\mathscr{K}_{V}\right|$ vary rather gradually, but a further increase of $\omega$ causes $\left|\Lambda_{V}\right|$ and $\left|\mathscr{K}_{V}\right|$ to decrease steeply. The peak at the synchronisation frequency becomes extremely sharp at large $R$, and it occurs for other forms of excitation. A simple mathematical explanation was given by [57].

Figure 11 presents also the DNS results. The predictions by the finite- $R$ theory agree with the DNS results almost precisely at moderate Reynolds numbers, and approach the asymptotic calculations as $R \rightarrow \infty$. The improved large $R$ is satisfactory even at $R=O\left(10^{3}\right)$.

\subsubsection{The receptivity to streamwise velocity and temperature perturbations}

In this section, we consider the receptivity to the streamwise velocity and temperature perturbations. DNS again were carried out, in which the wall boundary condition for the streamwise velocity perturbation is

$$
(u, v, T)=\left(\epsilon_{w} \mathrm{e}^{-x^{2} / d^{2}} \cos (\omega t), 0, T_{w}\right),
$$

while for the temperature perturbation the wall boundary condition is

$$
(u, v, T)=\left(0,0, T_{w}+\epsilon_{w} \mathrm{e}^{-x^{2} / d^{2}} \cos (\omega t)\right),
$$

with $\epsilon_{w}=10^{-6}$ and $\triangle_{w} / \delta_{99}=2.0$. The resulting initial amplitudes are denoted by $\mathscr{A}_{U}$ and $\mathscr{A}_{T}$, respectively.

Figure 12 displays the dependence on $R$ of the scaled initial amplitude $R^{1 / 2}\left|\mathscr{A}_{U}\right|$ and $R^{1 / 2}\left|\mathscr{A}_{T}\right|$. The results follow the same trend as that in Fig. 10. As $R \rightarrow \infty, R^{1 / 2}\left|\mathscr{K}_{U}\right|$ and $R^{1 / 2}\left|\mathscr{K}_{T}\right|$ (red solid lines) approach, respectively, the constant values, shown as the black dot-dashed lines. The increase of $R^{1 / 2}\left|\mathscr{K}_{U}\right|$ and $R^{1 / 2}\left|\mathscr{K}_{T}\right|$ with $R$ is monotonic except when $\omega=0.185$. The dependence on $R$ and the non-monotonic feature are well predicted by the improved 

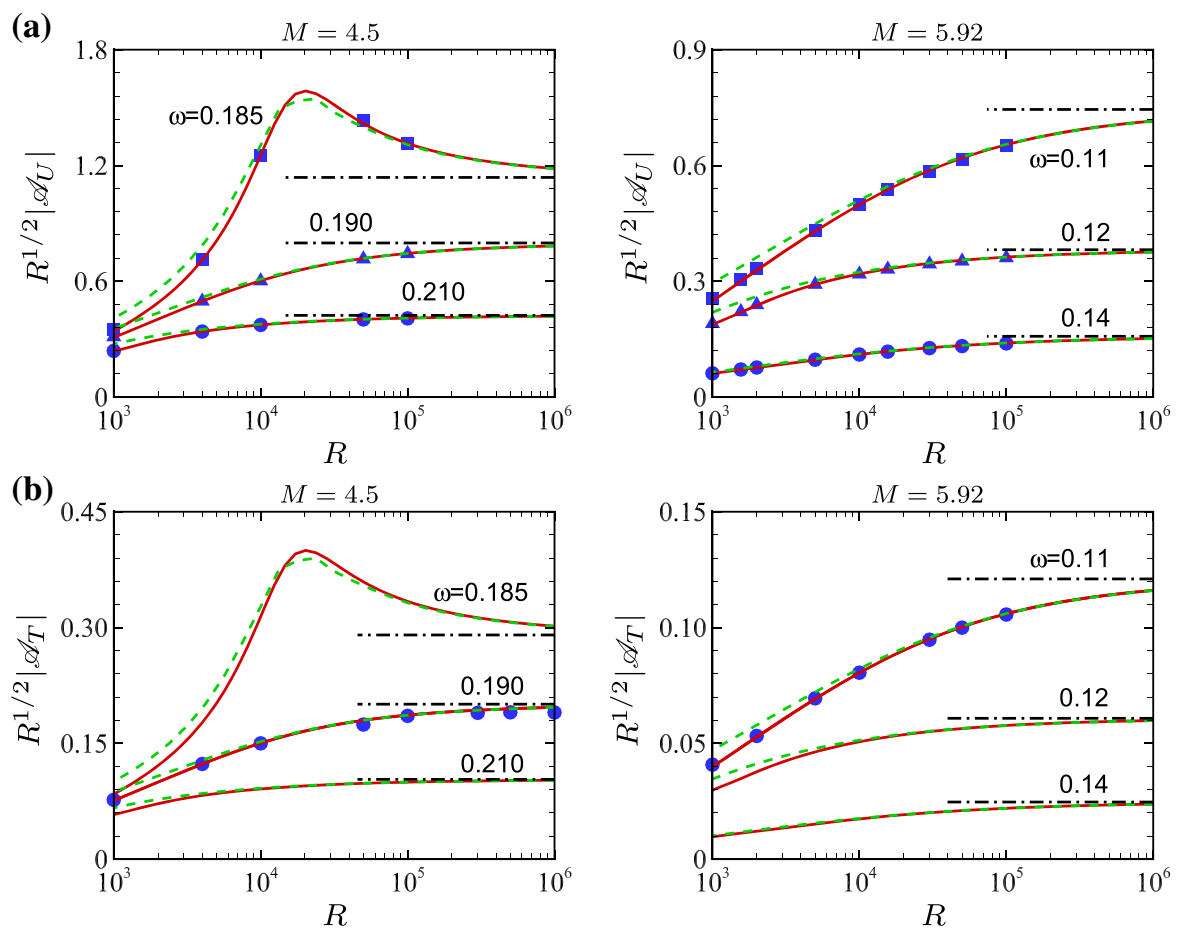

Fig. 12 The dependence on $R$ of the rescaled initial amplitude $R^{1 / 2}\left|\mathscr{A}_{U}\right|$ (a) and $R^{1 / 2}\left|\mathscr{A}_{T}\right|$ (b) for the streamwise velocity and temperature perturbations under conditions 1 (left column) and 2 (right column), where the line types are the same as those in Fig. 10

large- $R$ approach. The agreement between results given by the finite- $R$, improved large- $R$ and DNS approaches is quite satisfactory even when $R$ is fairly moderate, e.g. $R=10^{3}$. It is also verified that as $R$ increases, $\left|\mathscr{K}_{U}\right|$ and $\left|\mathscr{K}_{T}\right|$ obtained from the finite- $R$ decrease as $R^{-1 / 2}$, which confirms the scaling represented by (39). The boundary layer is found to be most sensitive to blowing/suction and least sensitive to temperature perturbation at the wall temperatures considered.

Figure 13 displays the dependence on $\omega$ of the normalised initial amplitude $R^{1 / 2}\left|\mathscr{A}_{U}\right|$ and $R^{1 / 2}\left|\mathscr{A}_{T}\right|$ for two different Reynolds numbers. Again, the results given by the finite- $R$ and DNS overlap for all the frequencies and Reynolds numbers considered, and the former approach the asymptotic calculations as $R \rightarrow \infty$. The prediction by the improved large- $R$ approach achieves satisfactory accuracy even for $R=O\left(10^{3}\right)$. For condition 2 , the receptivity coefficient at large $R$ exhibits a sharp peak at the synchronisation frequency. Increase of $R$ leads to a stronger receptivity.

In order to check the validity and accuracy of our results, Fig. 14 compares our $R^{1 / 2}\left|\Lambda_{U} / \Lambda_{V}\right|$ and $R^{1 / 2}\left|\Lambda_{T} / \Lambda_{V}\right|$ with those obtained by [73]. The latter are extracted from Fig. 23 in [73], which displays normalised amplitudes of the pressure fluctuations for three forms of wall perturbations: streamwise velocity, blowing/suction and temperature, referred to as cases $A, B$ and $C$, respectively.

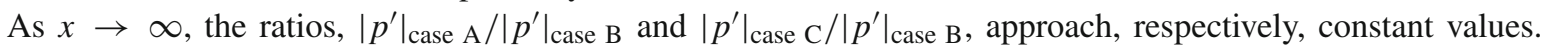
They can, after taking into account the amplitudes of the forcing, be converted, respectively, to $\left|\mathscr{A}_{U}\right| /\left|\mathscr{A}_{V}\right|$ and $\left|\mathscr{A}_{T}\right| /\left|\mathscr{A}_{V}\right|$ defined by (39). Furthermore, with the distribution functions of the wall disturbances given by Eq. (28), Eq. (34) and Eq. (36) in [73], we calculated their Fourier transforms $\hat{F}(\alpha)$, and then using (13) and (38), the scaled receptivity coefficient ratios, $R^{1 / 2}\left|\Lambda_{U} / \Lambda_{V}\right|$ and $R^{1 / 2}\left|\Lambda_{T} / \Lambda_{V}\right|$, were obtained. There is a good agreement between our results and those of [73].Thus, the results verify the quantitative connections between the three forms of wall perturbations: the receptivity coefficients for the streamwise velocity and temperature perturbations are $O\left(R^{-1 / 2}\right)$ smaller than that for the blowing/suction, with the ratios of the former to the latter taking fixed values. 

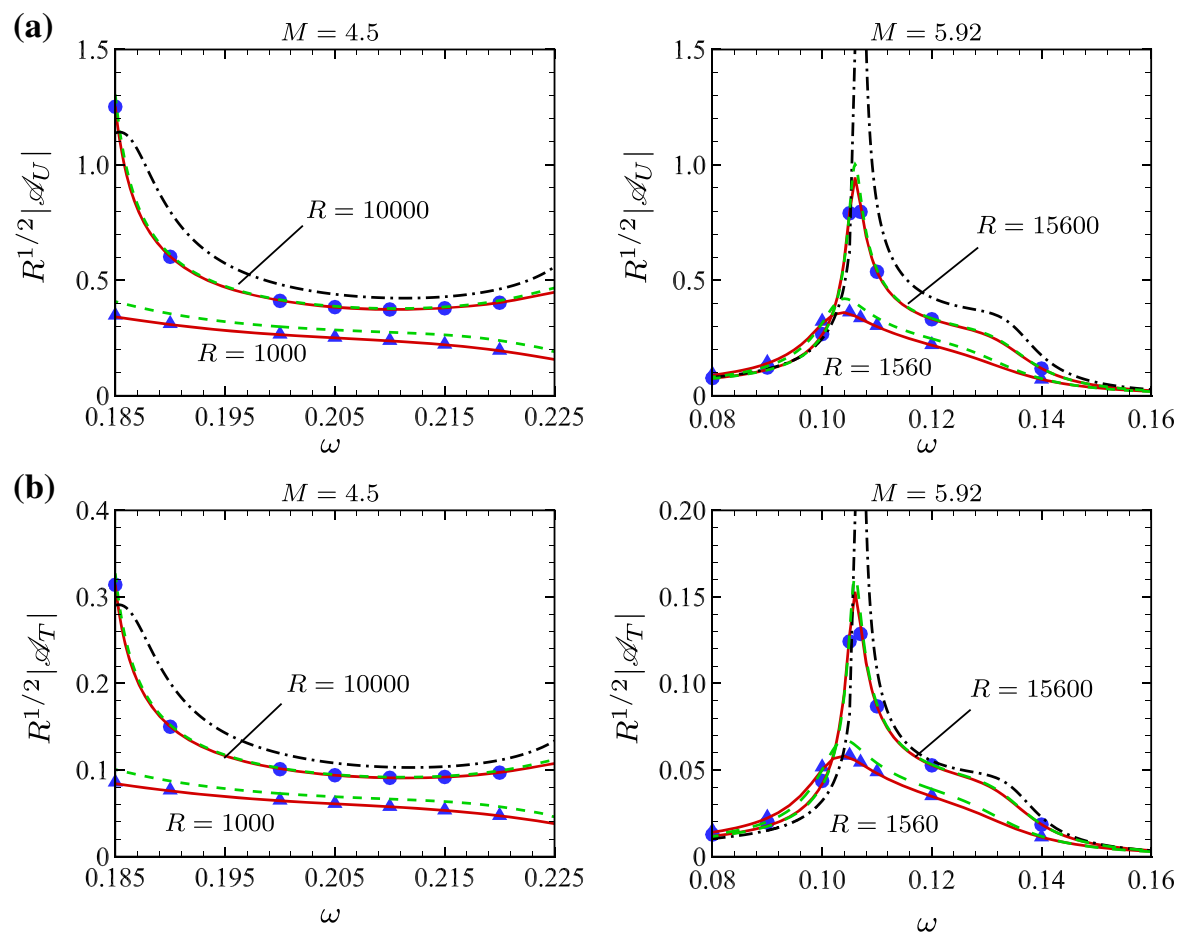

Fig. 13 The dependence on $\omega$ of the rescaled initial amplitude $R^{1 / 2}\left|\mathscr{A}_{U}\right|$ (a) and $R^{1 / 2}\left|\mathscr{A}_{T}\right|$ (b) for the streamwise velocity and temperature perturbations under conditions 1(left column) and 2(right column), where the line types are the same as those in Fig. 10
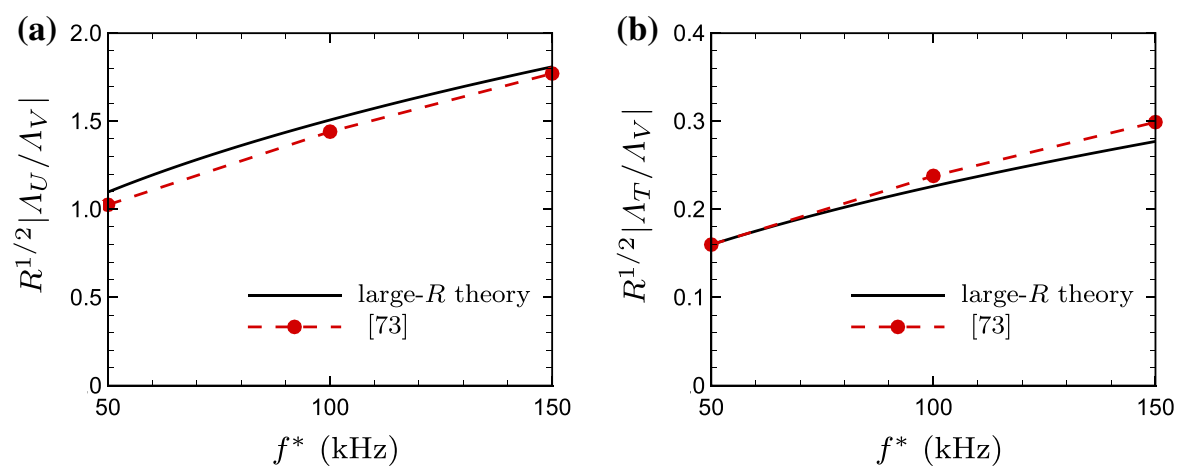

Fig. 14 The dependence on $f^{*}$ of the ratio $R^{1 / 2}\left|\Lambda_{U} / \Lambda_{V}\right|$ (a) and $R^{1 / 2}\left|\Lambda_{T} / \Lambda_{V}\right|$ (b), where the solid (black) lines, dashed (red) lines with symbols represent the results predicted by (38) and [73], respectively

\subsubsection{The receptivity for different wall temperatures}

According to (39), the ratios of the receptivity coefficients for the streamwise velocity and temperature perturbations to that for blowing/suction depend on the mean wall temperature $T_{w}$. In this section, we focus on the receptivity for different $T_{w}$.

Figure 15 compares the initial amplitude obtained by the finite- $R$, improved large- $R$ and large- $R$ approach for different $T_{w}$. The frequencies shown in the plots are in the band of the second-mode instability. For the streamwise velocity and temperature perturbations, the receptivity coefficients are normalised by $R^{1 / 2}$. For $T_{w}=4.4(M=4.5)$ 

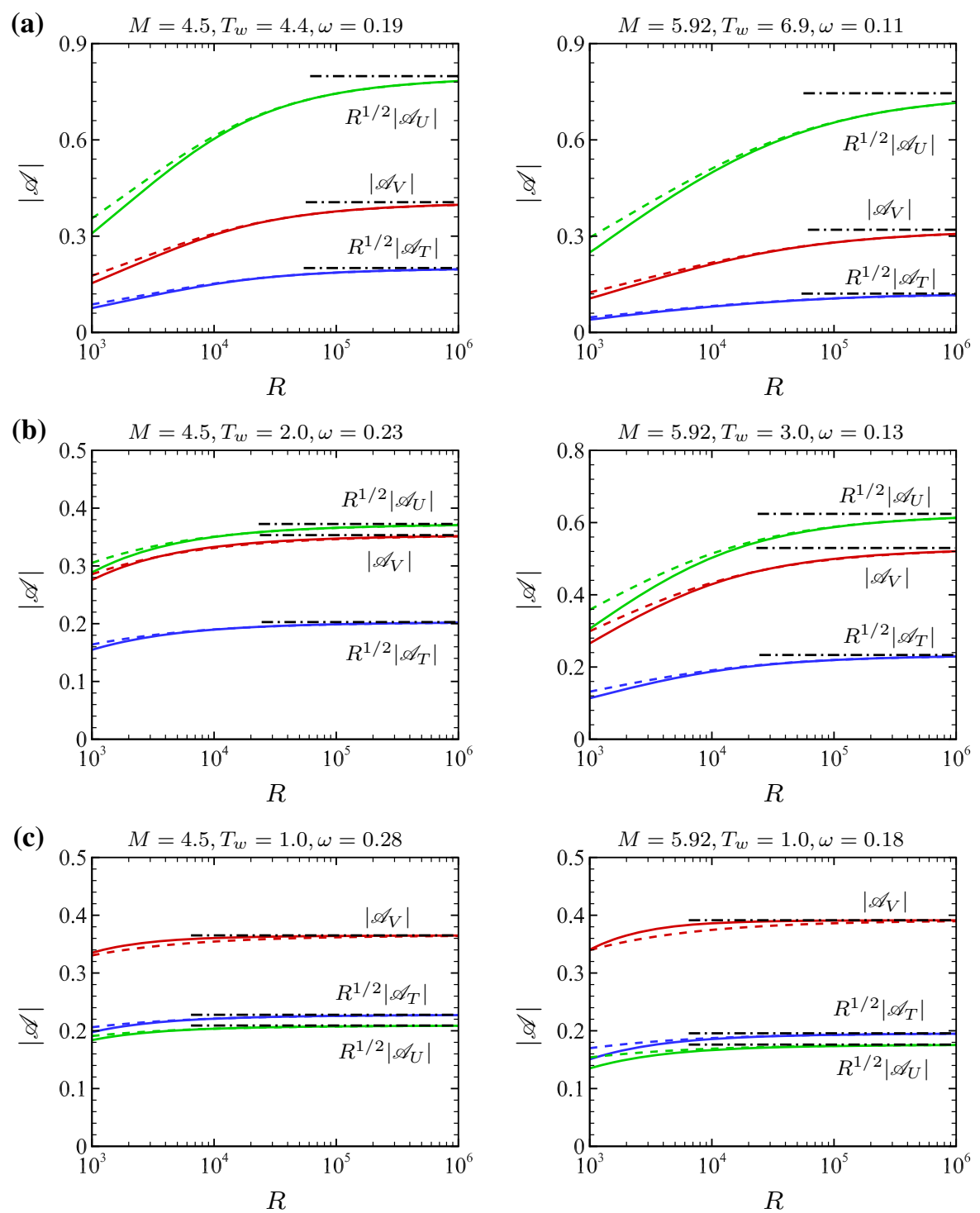

Fig. 15 The initial amplitude for three forms of wall perturbations at $T_{w}=4.4(M=4.5)$ and $T_{w}=6.9(M=5.92)(\mathbf{a}), T_{w}=2.0$ $(M=4.5)$ and $T_{w}=3.0(M=5.92)(\mathbf{b})$, and $T_{w}=1.0(\mathbf{c})$ under conditions 1 (left column) and 2 (right column). Solid lines: finite- $R$ theory; dashed lines: improved large- $R$ theory; dot-dashed lines: large- $R$ theory

and $T_{w}=6.9(M=5.92)$, good agreement is achieved between the finite- $R$ and improved large- $R$ approaches when $R \leq 3000$. As the wall is cooled, the receptivity becomes less sensitive to the Stokes-layer correction. Indeed, for the case of $T_{w}=1$, the correction is so slight that the leading-order inviscid theory predicts the receptivity coefficient rather accurately. It is worth noting that at both Mach numbers considered, cooling the wall reduces the receptivity to the streamwise velocity perturbation, but hardly influences that to the wall temperature perturbation (assumed to have a fixed magnitude relative to the free-stream temperature). As a result, the boundary layer changes from being more receptive to the streamwise velocity perturbation at $T_{w}=4.4(M=4.5)$ and $T_{w}=6.9(M=5.92)$ to being (marginally) more receptive to the wall temperature at $T_{w}=1$. At $M=4.5$, the receptivity to the blowing/suction is slightly affected by wall cooling, but is enhanced at $M=5.92$, 

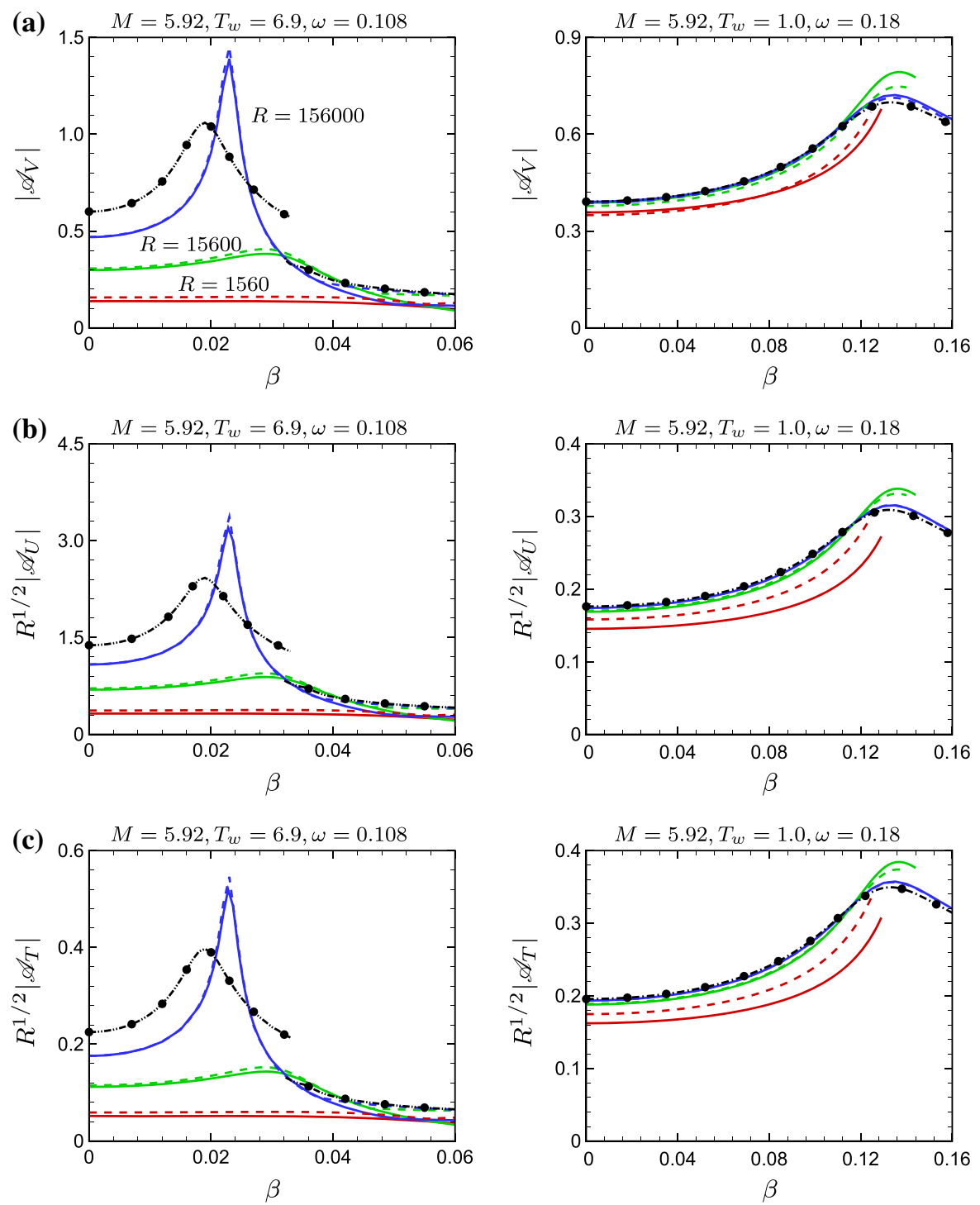

Fig. 16 The variation with the spanwise wavenumber $\beta$ of the initial amplitude of 3D instability mode under condition 2 for blowing/suction (a), streamwise velocity perturbation (b) and temperature perturbation (c). Solid lines: finite- $R$ approach; (black) dot-dashed lines with dots: large- $R$ approach; dashed lines: improved large- $R$ approach. The red, green and blue lines stand for the cases of $R=1560$, 15600 and 156000 , respectively

\subsubsection{The receptivity to three-dimensional wall perturbations}

Figure 16 shows the receptivity to three-dimensional wall perturbations for the base flow under condition 2 and the frequencies being the same as those in Fig. 8. For $T_{w}=6.9$, the initial amplitude obtained by the large- $R$ approach is represented by two curves, corresponding to the first and second instability modes for larger and smaller $\beta$, respectively. The receptivity of the first mode is much weaker, and decreases monotonically with $\beta$. The purely inviscid theory indicates that the receptivity of the second-mode peaks at $\beta \approx 0.02$, which corresponds to the near synchronisation wavenumber as is indicated in Fig. 8. Interestingly, when the impact of $R$ is taken into account, either by the finite- $R$ or by the improved large- $R$ approach, the peak receptivity of the second mode becomes sharper for a range of large Reynolds numbers. Although not shown, with further increase of $R$, the peak subdues and becomes broader, approaching the inviscid prediction. On the other hand, the peak is reduced and finally 
disappears at moderate Reynolds numbers. Again, the improved large- $R$ approach is able to predict the receptivity with reasonable accuracy even when $R$ is about a few thousands. For $T_{w}=1.0$, the purely inviscid and improved large- $R$ theories indicate that the receptivity of the second-mode peaks at $\beta \approx 0.13$. This peak remains for large values of $R$.

\section{Summary and discussion}

In this paper, we have investigated the receptivity of inviscid first and second modes in a supersonic boundary layer to time-periodic streamwise localised wall perturbations, namely, blowing/suction, streamwise velocity perturbation and temperature perturbation, all introduced by a forcing slot on the flat plate. Three complementary approaches have been employed, including a finite-Reynolds-number theory [37,38,57], and the large-Reynolds-number asymptotic theory and DNS, which is performed in order to assess the accuracy of the former two approaches.

Comparisons with the DNS results indicate that the finite-Reynolds-number theory predicts the receptivity at all Reynolds numbers with high accuracy. However, this methodology and DNS do not probe into the detailed physical process of the excitation by the streamwise velocity and temperature perturbations, nor could they explain why the receptivity to these perturbations is much weaker than that to blowing/suction. In order to shed light on these issues, the high-Reynolds-number approach was adopted, which shows that the perturbation acquires an asymptotic structure consisting of two layers: the inviscid main part of the boundary layer and a viscous Stokes layer adjacent to the wall. The analysis of the viscous and compressible motion in the latter shows that the streamwise velocity and temperature perturbations both generate $O\left(R^{-1 / 2} \epsilon_{w}\right)$ transpiration velocities to force upon the main layer. These transpiration velocities are much smaller than $O\left(\epsilon_{w}\right)$ blowing/suction velocity in the case of blowing/suction, but it transpires that the receptivity problems to all three forms of wall perturbations are the same when described in the large- $R$ asymptotic framework. Mathematically, they are governed by the compressible Rayleigh equation subject to a forcing in the form of the equivalent transverse velocity, in contrast to the finite- $R$ framework, in which a different wall boundary condition is imposed for each case. This equivalence offers useful insight into the receptivity mechanism, and provides the quantitative connections between the three cases: the receptivity coefficients for the streamwise velocity and temperature perturbations are $O\left(R^{-1 / 2}\right)$ smaller than that for the blowing/suction, with the ratios of the former to the latter taking fixed values.

The leading-order large-Reynolds-number approach predicts the receptivity coefficients accurately if the Reynolds number is set to be sufficiently large. However, at moderate Reynolds numbers, appreciable error exists because the high-order terms neglected are no longer small. The main correction is the $O\left(R^{-1 / 2}\right)$ transverse velocity from the Stokes layer. This affects the dispersion relation of the instability, which may in turn influence the receptivity calculation quantitatively at moderate Reynolds numbers. An improved large-Reynolds-number theory for instability follows from accounting for this effect simply by replacing the impermeability boundary condition by an appropriate impedance condition. When this 'impedance' effect of the Stokes layer is included along with the equivalent forcing, the predicted receptivity coefficients turned to be accurate at moderate Reynolds numbers $\left(R=O\left(10^{3}\right)\right)$ of practical interest. The improved theory captures satisfactorily the Reynolds-number dependence, which may be non-monotonic, as well as the strong excitation close to the synchronisation point, which is a finite-Reynolds-number phenomenon. By combining finite-Reynolds-number calculations, DNS and the largeReynolds-number asymptotic approach including the improved version, the present study has led to fairly thorough understanding and characterisation of the local receptivity of Mack modes in a supersonic boundary layer. On the balance of the computational cost, detailed insight provided and quantitative accuracy, it may be argued that the improved large-Reynolds-number approach stands as the most satisfactory methodology.

In a broader sense, the problem studied in the present paper exemplifies the fact that systematical asymptotic analysis, ad hoc modelling and DNS serve rather different purposes, and so they are all necessary tools and complement to each other. Insofar as the rule-based science is not to be replaced completely by data bank, and scientific quest for cause-and-effect is not to be abandoned completely for empirical correlations or graphical animations, asymptotic analysis will remain indispensable however powerful and cheap computing will become. 
When it comes to solving multi-scale, multi-physics problems, asymptotic framework is the one in which relevant physical mechanisms can be revealed, quantified and stated.

Acknowledgements This work was supported by NSFC (Grant Nos. 91952202 and 11772224).

Open Access This article is licensed under a Creative Commons Attribution 4.0 International License, which permits use, sharing, adaptation, distribution and reproduction in any medium or format, as long as you give appropriate credit to the original author(s) and the source, provide a link to the Creative Commons licence, and indicate if changes were made. The images or other third party material in this article are included in the article's Creative Commons licence, unless indicated otherwise in a credit line to the material. If material is not included in the article's Creative Commons licence and your intended use is not permitted by statutory regulation or exceeds the permitted use, you will need to obtain permission directly from the copyright holder. To view a copy of this licence, visit http://creativecommons.org/licenses/by/4.0/.

\section{Appendix A: Composite solution for the eigenfunction}

In the large-Reynolds-number asymptotic theory, the solution for the inviscid mode is given primarily in the inviscid main layer but a viscous solution is obtained in the Stokes layer in order to satisfy the no-slip condition at the wall. Using those solutions, a composite solution uniformly valid in the entire boundary layer can be constructed.

In the main deck, after obtaining $\hat{v}_{I}$ and $\hat{p}_{I}$ by solving numerically the eigenvalue problem, (40) with $\hat{v}_{I}(0)=0$ and $\hat{p}_{I} \rightarrow 0$ as $y \rightarrow \infty$, it follows from the linearised Euler equations that solution for $\hat{u}_{I}, \hat{w}_{I}, \hat{\rho}_{I}$ and $\hat{\theta}_{I}$,

$$
\left\{\begin{array}{l}
\hat{u}_{I}=\left(-\mathrm{i} \alpha T_{B} \hat{p}_{I}-U_{B}^{\prime} \hat{v}_{I}\right) / S_{0}, \quad \hat{w}_{I}=-\mathrm{i} \beta T_{B} \hat{p}_{I} / S_{0}, \\
\hat{\rho}_{I}=T_{B}^{\prime} \hat{v}_{I} /\left(T_{B}^{2} S_{0}\right)+M^{2} \hat{p}_{I} / T_{B}, \quad \hat{\theta}_{I}=(\gamma-1) M^{2} T_{B} \hat{p}_{I}-T_{B}^{\prime} \hat{v}_{I} / S_{0} .
\end{array}\right.
$$

In the Stokes layer, the solution can be written as

$(\hat{U}, \hat{w})=(\alpha, \beta) T_{w} \omega^{-1}\left(1-\mathrm{e}^{(-\mathrm{i} \omega)^{1 / 2} Y}\right) \hat{p}_{I}(0)$,

where the Stokes-layer coordinate $Y$ is defined by (18).

Using the main solution (46) and Stokes-layer solution (47), the composite solution, denoted by $\left(\widehat{u}_{c}, \widehat{w}_{c}\right)$, is constructed as

$$
\left\{\begin{array}{l}
\widehat{u}_{c}=\hat{u}_{I}(y)-\alpha T_{w} \omega^{-1} \mathrm{e}^{(-\mathrm{i} \omega)^{1 / 2} Y} \hat{p}_{I}(0), \\
\widehat{w}_{c}=\hat{w}_{I}(y)-\beta T_{w} \omega^{-1} \mathrm{e}^{(-\mathrm{i} \omega)^{1 / 2} Y} \hat{p}_{I}(0) .
\end{array}\right.
$$

For the improved large-Reynolds-number asymptotic theory, the Stokes-layer correction to the main-layer solution is taken into account simply by replacing the impermeability condition by (41). The composite solution, denoted by $\left(\widehat{u}_{c}, \widehat{w}_{c}\right)$, is reconstructed as

$$
\left\{\begin{array}{l}
\widehat{u}_{c}=\hat{u}_{I}(y)-\alpha T_{w} \omega^{-1} \mathrm{e}^{(-\mathrm{i} \omega)^{1 / 2} Y} \hat{p}_{I}(0)+\left(R / C_{w}\right)^{-1 / 2} \frac{U_{B}^{\prime}(0)}{\mathrm{i} \omega} H_{\mathrm{V}} \hat{p}_{I}(0), \\
\widehat{w}_{c}=\hat{w}_{I}(y)-\beta T_{w} \omega^{-1} \mathrm{e}^{(-\mathrm{i} \omega)^{1 / 2} Y} \hat{p}_{I}(0),
\end{array}\right.
$$

where

$H_{\mathrm{V}}=\frac{\mathrm{i}(\gamma-1) \omega M^{2}}{(-\mathrm{i} \omega P r)^{1 / 2}} \mathrm{e}^{(-\mathrm{i} \omega P r)^{1 / 2} Y}+\frac{\left(k^{2}+\beta^{2}\right) T_{w}}{(-\mathrm{i} \omega)^{3 / 2}} \mathrm{e}^{(-\mathrm{i} \omega)^{1 / 2} Y}$.

\section{References}

1. Kimmel RL (2003) Aspects of hypersonic boundary-layer transition control. AIAA Paper 2003-772

2. Reshotko E (2008) Transition issues for atmospheric entry. J Spacecr Rockets 45:161-164

3. Reed HL, Saric WS, Arnal D (1996) Linear stability theory applied to boundary layers. Annu Rev Fluid Mech 28:389-428 
4. Hall P (1990) Görtler vortices in growing boundary layers: the leading edge receptivity problem, linear growth and the nonlinear breakdown stage. Mathematika 37:151-189

5. Saric WS (1994) Görtler vortices. Ann Rev Fluid Mech 26:379-409

6. Hall P, Malik MR, Poll DIA (1984) On the stability of an infinite swept attachment line boundary layer. Proc R Soc Lond A 395:229-245

7. Reed HL, Saric WS (1989) Stability of three-dimensional boundary layers. Ann Rev Fluid Mech 21:235-284

8. Gregory N, Stuart JT, Walker WS (1955) On the stability of three-dimensional boundary layers with application to the flow due to a rotating disk. Philos Trans R Soc Lond A 248:155-199

9. Butler A, Wu X (2018) Stationary crossflow vortices near the leading edge of three-dimensional boundary layers: the role of non-parallelism and excitation by surface roughness. J Fluid Mech 845:93-140

10. Kachanov YS (1994) Physical mechanisms of laminar-boundary-layer transition. Annu Rev Fluid Mech 26:411-482

11. Morkovin MV (1969) Critical evaluation of transition from laminar to turbulent shear layers with emphasis on hypersonically travelling bodies. Tech. Rep. AFFDL-TR pp. 68-149

12. Wu X (2019) Nonlinear theories for shear flow instabilities: physical insights and practical implications. Annu Rev Fluid Mech 51:451-485

13. Goldstein ME, Hultgren LS (1989) Boundary-layer receptivity to long-wave free-stream disturbances. Ann Rev Fluid Mech 21:137166

14. Saric WS, Reed HL, Kerschen EJ (2002) Boundary-layer receptivity to freestream disturbances. Ann Rev Fluid Mech 34:291-319

15. Goldstein ME (1985) Scattering of acoustic waves into Tollmien-Schlichting waves by small streamwise variations in surface geometry. J Fluid Mech 154:509-529

16. Ruban AI (1984) On Tollimien-Schlichting wave generation by sound (in Russian). Izv. Akad. Nauk SSSR Mekh. Zhidk. Gaza 5:44-52 (translation in Fluid Dyn 19:709-716)

17. Heisenberg W (1924) Über stabilität und turbulenz von flüssigkeitsströmen. Annu Phys 74:577-627

18. Schlichting H (1993) Zur enstehung der turbulenz bei der plattenströmung. ZAMM Z Angew Math Mech 13:171-174

19. Tollmien W (1929) Über die entstehung der turbulenz. Nachr. Ges. Wiss. Görttingen, Math Phys Klasse. pp 21-44. English Translation in NACA-TM-609 (1931)

20. Lin CC (1946) On the stability of two-dimensional parallel flows. Part III. Stability in a viscous fluid. Q Appl Math 3(4):277-301

21. Lin CC (1955) The theory of hydrodynamics stability. Cambridge University Press, Cambridge

22. Smith FT (1979) On the non-parallel flow stability of the Blasius boundary layer. Proc R Soc Lond A 366:91-109

23. Blackaby ND, Cowley SJ, Hall P (1993) On the instability of hypersonic flow past a flat plate. J Fluid Mech 247:369-416

24. Smith FT (1989) On the first-mode instability in subsonic, supersonic or hypersonic boundary layers. J Fluid Mech 198:127-153

25. Smith FT, Brown SN (1990) The inviscid instability of a Blasius boundary layer at large values of the Mach number. J Fluid Mech 219:499-518

26. Choudhari M (1995) Long-wavelength asymptotics of unstable crossflow modes, including the effect of surface curvature. Proc R Soc Lond A 451:515-541

27. Goldstein ME (1983) The evolution of Tollmien-Schlichting waves near a leading edge. J Fluid Mech 127:59-81

28. Haddad OM, Corke TC (1998) Boundary layer receptivity to free-stream sound on parabolic bodies. J Fluid Mech 368:1-26

29. Hammerton PW, Kerschen EJ (1996) Boundary-layer receptivity for a parabolic leading edge. J Fluid Mech 310:243-267

30. Duck PW, Ruban AI, Zhikharev CN (1996) The generation of Tollmien-Schlichting waves by free-stream turbulence. J Fluid Mech 312:341-371

31. Wu X (2001) On local boundary-layer receptivity to vortical disturbances in the free stream. J Fluid Mech 449:373-393

32. Wu X (2001) Receptivity of boundary layers with distributed roughness to vortical and acoustic disturbances: a second-order asymptotic theory and comparison with experiments. J Fluid Mech 431:91-133

33. Wu X (1999) Generation of Tollmien-Schlichting waves by convecting gusts interacting with sound. J Fluid Mech 397:285-316

34. De Tullio N, Ruban AI (2015) A numerical evaluation of the asymptotic theory of receptivity for subsonic compressible boundary layers. J Fluid Mech 771:520-546

35. Ruban AI, Bernots T, Fryce D (2013) Receptivity of the boundary layer to vibrations of the wing surface. J Fluid Mech 723:480-528

36. Ruban AI, Bernots T, Kravtsova MA (2016) Linear and nonlinear receptivity of the boundary layer in transonic flows. J Fluid Mech 786:154-189

37. Choudhari M, Streett CL (1992) A finite Reynolds-number approach for the prediction of boundary-layer receptivity in localized regions. Phys Fluids A 4(11):2495-2514

38. Crouch JD (1992) Localized receptivity of boundary layers. Phys Fluids A 4:1408

39. Fedorov AV (2011) Transition and stability of high-speed boundary layers. Annu Rev Fluid Mech 43:79-95

40. Zhong X, Wang X (2012) Direct numerical simulation on the receptivity, instability, and transition of hypersonic boundary layers. Annu Rev Fluid Mech 44(1):527-561

41. Mack LM (1984) Boundary-layer linear stability theory. AGARD Rep. 709

42. Mack LM (1987) Review of linear compressible stability theory. In: Dwoyer DL, Hussaini MY (eds) Stability of time dependent and spatially varying flows. Springer, New York, pp 164-187

43. Fedorov AV (2003) Receptivity of a high-speed boundary layer to acoustic disturbances. J Fluid Mech 491:101-129

44. Fedorov AV, Khokhlov AP (1991) Excitation of unstable modes in a supersonic boundary layer by acoustic waves. Fluid Dyn 9:456-467 
45. Fedorov AV, Khokhlov AP (2001) Prehistory of instability in a hypersonic boundary layer. Theor Comput Fluid Dyn 14:359-375

46. Maslov AA, Shiplyuk AN, Sidorenko AA, Arnal D (2001) Leading-edge receptivity of a hypersonic boundary layer on a flat plate. J Fluid Mech 426:73-94

47. Balakumar P (2009) Receptivity of a supersonic boundary layer to acoustic disturbances. AIAA J 47(5):1069-1078

48. Balakumar P, Kegerise MA (2010) Receptivity of hypersonic boundary layers over straight and flared cones. AIAA Paper 2010-1065

49. Ma Y, Zhong X (2003) Receptivity of a supersonic boundary layer over a flat plate. Part 1 . Wave structures and interactions. J Fluid Mech 488:31-78

50. Ma Y, Zhong X (2003) Receptivity of a supersonic boundary layer over a flat plate. Part 2. Receptivity to free-stream sound. J Fluid Mech 488:79-121

51. Ma Y, Zhong X (2005) Receptivity of a supersonic boundary layer over a flat plate. Part 3. Effects of different types of free-stream disturbances. J Fluid Mech 532:63-109

52. Zhong X (2001) Leading-edge receptivity to free stream disturbance waves for hypersonic flow over a parabola. J Fluid Mech 441:315-367

53. Ricco P, Wu X (2007) Response of a compressible laminar boundary layer to free-stream vortical disturbances. J Fluid Mech 587:97-138

54. Goldstein ME, Ricco P (2018) Non-localized boundary layer instabilities resulting from leading edge receptivity at moderate supersonic Mach numbers. J Fluid Mech 838:435-477

55. Liu Y, Dong M, Wu X (2020) Generation of first Mack modes in supersonic boundary layers by slow acoustic waves interacting with streamwise isolated wall roughness. J Fluid Mech 888:A10

56. Hernández CG, Wu X (2019) Receptivity of supersonic boundary layers over smooth and wavy surfaces to impinging slow acoustic waves. J Fluid Mech 872:849-888

57. Dong M, Liu Y, Wu X (2020) Receptivity of inviscid modes in supersonic boundary layers due to scattering of free-stream sound by localised wall roughness. J Fluid Mech 896:A23

58. Duck PW, Lasseigne DG, Hussaini MY (1995) On the interaction between the shock wave attached to a wedge and freestream disturbances. Theor Comput Fluid Dyn 7(2):119-139

59. Duck PW, Lasseigne DG, Hussaini MY (1997) The effect of three-dimensional freestream disturbances on the supersonic flow past a wedge. Phys Fluids 9(2):456-467

60. Maslov AA, Mironov SG, Kudryavtsev AN, Poplavskaya TV, Tsyryulnikov IS (2010) Wave processes in a viscous shock layer and control of fluctuations. J Fluid Mech 650:81-118

61. Mckenzie JF, Westphal KO (1968) Interaction of linear waves with oblique shock waves. Phys Fluids 11:2350-2362

62. Balakumar P (2005) Transition in a supersonic boundary layer due to acoustic disturbances. AIAA Paper 2005-96

63. Wan B, Luo J, Su C (2018) Response of a hypersonic blunt cone boundary layer to slow acoustic waves with assessment of various routes of receptivity. Appl Math Mech 39:1643-1660

64. Wan B, Su C, Chen J (2020) Receptivity of a hypersonic blunt cone: role of disturbances in entropy layer. AIAA J 4:1-8

65. Cowley SJ, Hall P (1990) On the instability of hypersonic flow past a wedge. J Fluid Mech 214:17-42

66. Qin F, Wu X (2016) Response and receptivity of the hypersonic boundary layer past a wedge to free-stream acoustic, vortical and entropy disturbances. J Fluid Mech 797:874-915

67. Gaster M (1965) On the generation of spatially growing waves in a boundary layer. J Fluid Mech 22:433-441

68. Tumin AM, Fedorov AV (1984) Instability wave excitation by a localized vibrator in the boundary layer. J Appl Mech Tech Phys 25:867-873

69. Bodonyi RJ, Duck PW (1992) Boundary-layer receptivity due to a wall suction and control of Tollmien-Schlichting waves. Phys Fluids A 4:1206-1214

70. Terent'ev ED (1981) Linear problem of a vibrator in subsonic boundary layer. Prikl Mat Mekh 45:1049-1055 (translation in Appl Math Mech 45:791-795)

71. Terent'ev ED (1984) The linear problem of a vibrator performing harmonic oscillations at supercritical frequencies in a subsonic boundary layer. PMM USSR 48:184-191

72. Fedorov AV, Khokhlov AP (2002) Receptivity of hypersonic boundary layer to wall disturbances. Theor Comput Fluid Dyn $15: 231-254$

73. Wang X, Zhong X (2009) Effect of wall perturbations on the receptivity of a hypersonic boundary layer. Phys Fluids $21: 044101$

74. Malik M (1990) Finite difference solution of the compressible stability eigenvalue problem. NASA Tech Rep 16572(86):376-413

75. Dong M, Zhou H (2013) A simulation on bypass transition and its key mechanism. Sci China Phys Mech Astron 56:775-784

76. Qin H, Dong M (2016) Boundary-layer disturbances subjected to free-stream turbulence and simulation on bypass transition. Appl Math Mech 37:967-986

77. Poinsot TJ, Lele SK (1992) Boundary conditions for direct simulations of compressible viscous flows. J Comput Phys 101:104-129

78. Fong K, Wang X, Zhong X (2014) Numerical simulation of roughness effect on the stability of a hypersonic boundary layer. Comput Fluids $96: 350-367$

79. Fong K, Wang X, Zhong X (2015) Parametric study on stabilization of hypersonic boundary-layer waves using 2-D surface roughness. AIAA Paper 2015-0837

Publisher's Note Springer Nature remains neutral with regard to jurisdictional claims in published maps and institutional affiliations. 